NASA/TM-2006-214112

\title{
Aligned and Unaligned Coherence: A New Diagnostic Tool
}

Jeffrey Hilton Miles

Glenn Research Center, Cleveland, Ohio 
Since its founding, NASA has been dedicated to the advancement of aeronautics and space science. The NASA Scientific and Technical Information (STI) Program Office plays a key part in helping NASA maintain this important role.

The NASA STI Program Office is operated by Langley Research Center, the Lead Center for NASA's scientific and technical information. The NASA STI Program Office provides access to the NASA STI Database, the largest collection of aeronautical and space science STI in the world. The Program Office is also NASA's institutional mechanism for disseminating the results of its research and development activities. These results are published by NASA in the NASA STI Report Series, which includes the following report types:

- $\quad$ TECHNICAL PUBLICATION. Reports of completed research or a major significant phase of research that present the results of NASA programs and include extensive data or theoretical analysis. Includes compilations of significant scientific and technical data and information deemed to be of continuing reference value. NASA's counterpart of peerreviewed formal professional papers but has less stringent limitations on manuscript length and extent of graphic presentations.

- TECHNICAL MEMORANDUM. Scientific and technical findings that are preliminary or of specialized interest, e.g., quick release reports, working papers, and bibliographies that contain minimal annotation. Does not contain extensive analysis.

- CONTRACTOR REPORT. Scientific and technical findings by NASA-sponsored contractors and grantees.
- CONFERENCE PUBLICATION. Collected papers from scientific and technical conferences, symposia, seminars, or other meetings sponsored or cosponsored by NASA.

- SPECIAL PUBLICATION. Scientific, technical, or historical information from NASA programs, projects, and missions, often concerned with subjects having substantial public interest.

- TECHNICAL TRANSLATION. Englishlanguage translations of foreign scientific and technical material pertinent to NASA's mission.

Specialized services that complement the STI Program Office's diverse offerings include creating custom thesauri, building customized databases, organizing and publishing research results ... even providing videos.

For more information about the NASA STI Program Office, see the following:

- Access the NASA STI Program Home Page at http://www.sti.nasa.gov

- E-mail your question via the Internet to help@sti.nasa.gov

- Fax your question to the NASA Access Help Desk at 301-621-0134

- Telephone the NASA Access Help Desk at 301-621-0390

- Write to:

NASA Access Help Desk

NASA Center for AeroSpace Information 7121 Standard Drive

Hanover, MD 21076 
NASA/TM-2006-214112

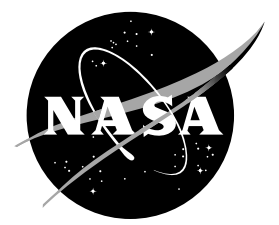

\section{Aligned and Unaligned Coherence: A New Diagnostic Tool}

Jeffrey Hilton Miles

Glenn Research Center, Cleveland, Ohio

Prepared for the

44th Aerospace Sciences Meeting and Exhibit

sponsored by the American Institute of Aeronautics and Astronautics

Reno, Nevada, January 9-12, 2006

National Aeronautics and

Space Administration

Glenn Research Center 
Trade names or manufacturers' names are used in this report for identification only. This usage does not constitute an official endorsement, either expressed or implied, by the National Aeronautics and Space Administration.

This work was sponsored by the Fundamental Aeronautics Program at the NASA Glenn Research Center.

Available from

NASA Center for Aerospace Information 7121 Standard Drive

Hanover, MD 21076
National Technical Information Service 5285 Port Royal Road Springfield, VA 22100 


\title{
Aligned and Unaligned Coherence: A New Diagnostic Tool
}

\author{
Jeffrey Hilton Miles \\ National Aeronautics and Space Administration \\ Glenn Research Center \\ Cleveland, Ohio 44135
}

\begin{abstract}
The study of combustion noise from turbofan engines has become important again as the noise from other sources like the fan and jet are reduced. A method has been developed to help identify combustion noise spectra using an aligned and unaligned coherence technique. When used with the well known 3 signal coherent power method and coherent power method it provides new information by separating tonal information from random process information. Examples are presented showing the underlying tonal structure which is buried under broadband noise and jet noise. The method is applied to data from a Pratt and Whitney PW4098 turbofan engine.
\end{abstract}

\section{Nomenclature}

$\delta(f) \quad$ the Dirac $\delta$ - function

$X \hat{(f)} \quad$ Expected value of the Fourier transform of $\mathrm{x}(\mathrm{t})$

$\hat{Y}(f) \quad$ Expected value of the Fourier transform of $\mathrm{y}(\mathrm{t})$

$\tau \quad$ integration variable

$a, b \quad$ amplitude factors

$B_{e} \quad$ resolution bandwidth, $\mathrm{Hz}, B_{e}=1 / T_{d}=r / N P=11.71875 \mathrm{~Hz}$

$c_{o} \quad$ turbofan engine to microphone sound propagation speed, $\frac{\mathrm{m} \text {. }}{\mathrm{sec} .}$

D delay, sec.

$d \quad$ time delay expressed as units of the sequence

$E[\quad]$ expected value of [

$E_{I} \quad$ confidence interval

$f \quad$ frequency, $\mathrm{Hz}$

$f_{c} \quad$ upper frequency limit, $f_{c}=1 / 2 \Delta t=r / 2, \mathrm{~Hz} .(24000 \mathrm{~Hz}$.

$F_{N} \quad$ the Fejer kernel

$G_{x x}(f)$ auto power spectral density function defined for non-negative frequencies only (one-sided)

$G_{x y}(f)$ cross power spectral density function defined for non-negative frequencies only (one-sided)

$h(\tau) \quad$ weighting function

$H(f)$ transfer function frequency response

$h_{11}$ auto-spectrum

$h_{12} \quad$ cross-spectrum

$I_{x x}^{k}(f, T)$ two sided auto-spectra periodogram, $E\left[S_{x x}(f, T, k)\right]$

$I_{x y}^{k}(f, T)$ two sided cross-spectra periodogram, $E\left[S_{x y}(f, T, k)\right]$

$j \quad$ positive imaginary square root of $-1, \sqrt{-1}$

$J_{x x}^{k}(f, T)$ one sided auto-spectra periodogram, $E\left[G_{x x}(f, T, k)\right]$

$J_{x y}^{k}(f, T)$ one sided cross-spectra periodogram, $E\left[G_{x y}(f, T, k)\right]$

$L_{y} \quad$ number of frequencies, $f_{c} / \Delta f=N P / 2$ ( 2048 )

$M \quad$ block size in points

$N$ total number of points in discrete sequence is $2 N-1$ 
N1CORR corrected rotor speed, rpm

$n_{1}, n_{2}$, and $n_{3}$ independent stationary Gaussian random time series

$n_{d} \quad$ number of disjoint (independent) segments used in spectra estimates of PW4098 test data, $n_{d}=$ $B_{e} T_{\text {total }}=234$

$n_{s} \quad$ number of segments/blocks

NP segment length, number of data points per segment (4096)

$P_{I} \quad$ probability confidence interval, percent

$R \quad$ distance from turbofan engine to microphone, $\mathrm{m}$.

$r \quad$ sample rate,samples/sec. (48000)

$\hat{R}_{x y}(\tau)$ estimated cross-correlation

$R_{x y}(\tau)$ cross-correlation

$R_{12}(s)$ cross-covariance function

$R_{\epsilon \epsilon}(s)$ auto-covariance function for $\epsilon_{t}$

$R_{x y} \quad$ cross-correlation function

$R_{y y} \quad$ auto-correlation function

$s \quad$ summation index for discrete spectra

$s_{F}(t) \quad$ output of the system

$S_{x y}(f)$ power spectral density function defined for both positive and negative frequencies (Two-Sided)

$t$ time, sec.

$T_{\text {total }}$ total record length,sec. $(\approx 20$ sec. $)$

$T_{d}(i)$ record length of segment $i, \frac{T_{\text {total }}}{n_{d}}, \frac{N P}{r}$, sec. $T_{d}=\frac{N P}{r}=4096 / 48000=0.08533 \mathrm{sec}$.

$u_{1}(t), u_{2}(t)$, and $u_{3}(t)$ core noise signals

$W_{s_{F} s_{F}}(f)$ coherent output power spectral function

$w(\tau \quad$ weighting function also known as a window function

$W(f) \quad$ Fourier transform of window function $\mathrm{w}(\mathrm{t})$

$x(t) \quad$ signal $\mathrm{x}$ time history

$x_{n}(t), y_{n}(t)$ and $z_{n}(t)$ noise time series

$y_{1}(t), y_{2}(t)$, and $y_{3}(t)$ external microphone signals

$y(t) \quad$ signal y time history

$\ell(t), m(t)$, and $n(t)$ uncorrelated noise signal from the jet

Subscripts

$i \quad$ running segment index

$x \quad$ signal $\mathrm{x}$

y signal $\mathrm{y}$

Symbols

$\alpha \quad$ noise time series mixing parameter

$\Delta f \quad$ frquency step, $1 / T_{d}, \mathrm{~Hz}$. (11.718)

$\Delta t \quad$ sampling interval, $1 / r(1 / 48000)$, sec.

$\gamma_{y_{1} y_{2}}(f)$ complex coherence function

$\hat{\gamma}_{r_{k} r_{\ell}}^{2}(f)$ estimated magnitude squared coherence (MSC) function

$\gamma_{x_{n} y_{n}}^{2}(f)$ magnitude squared coherence (MSC) function of noise, $\gamma_{x_{n} y_{n}}^{2}$

$\gamma_{x y}^{2}(f)$ magnitude squared coherence (MSC) function

[^] estimate of [ ]

$\omega \quad$ angular frequenchy, $2 \pi f$

$\tau \quad$ time displacement, sec.

$\tau_{1} \quad$ propagation time delay

$\tau_{o} \quad$ turbofan engine to microphone propagation time delay, $\frac{R}{c_{o}}$, sec., $0.1317=6323 / 48000$

$\ell(t), m(t)$, and $n(t)$ noise time series

Superscripts

* complex conjugate 


\section{Introduction}

\section{A. Motivation}

A key priority of the National Aeronautics and Space Administration (NASA) Aeronautics program is to achieve a noise reduction of $20 \mathrm{~dB}$ relative to 1997 state of the art noise reduction technology. Consequently, the objective of most outdoor aircraft turbofan engine noise test programs that NASA participates in is to evaluate the noise measurements to identify dominant sources. This report discusses new core noise diagnostic procedures developed to study correlated turbo machinery noise and correlated combustion noise.

\section{PW4098 Engine at C-11 Stand for EVNRC Phase 2 Tests}

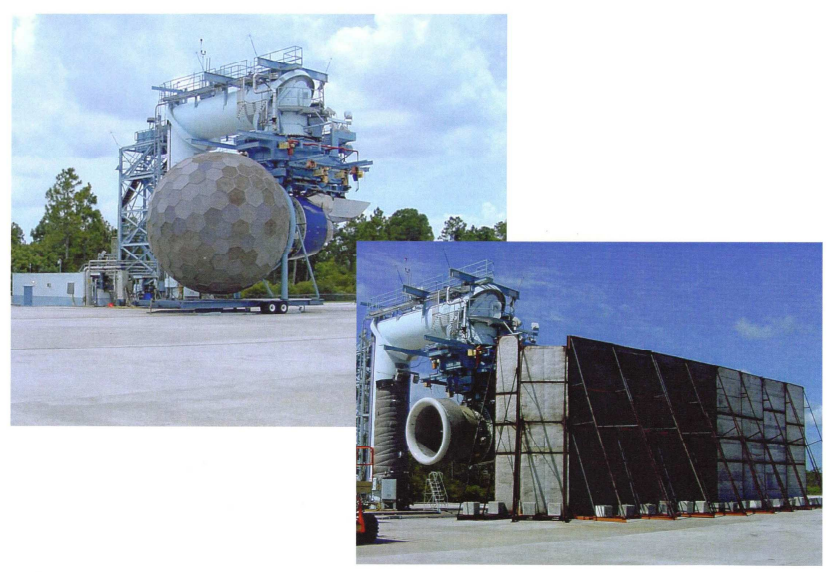

Figure 1. Pratt \& Whitney test stand C11, West Palm Beach Florida, with PW4098 engine and attached acoustic inflow control device also with and without aft acoustic barrier walls for EVNRC Phase 2 tests.

Flight data generally indicate more noise than would be expected from the theoretical predictions or model simulation tests. It is possible that internal noise sources (i.e. core noise) might become significant contributors to overall turbofan engine noise during takeoff or approach when the fan and jet noise are reduced because of forward velocity effects. Core noise consists of noise associated with combustor, compressor noise, and turbine noise. This paper discusses an extension of the three signal coherence technique to study compressor and turbine noise and an extension of the coherent output power technique to study combustor noise.

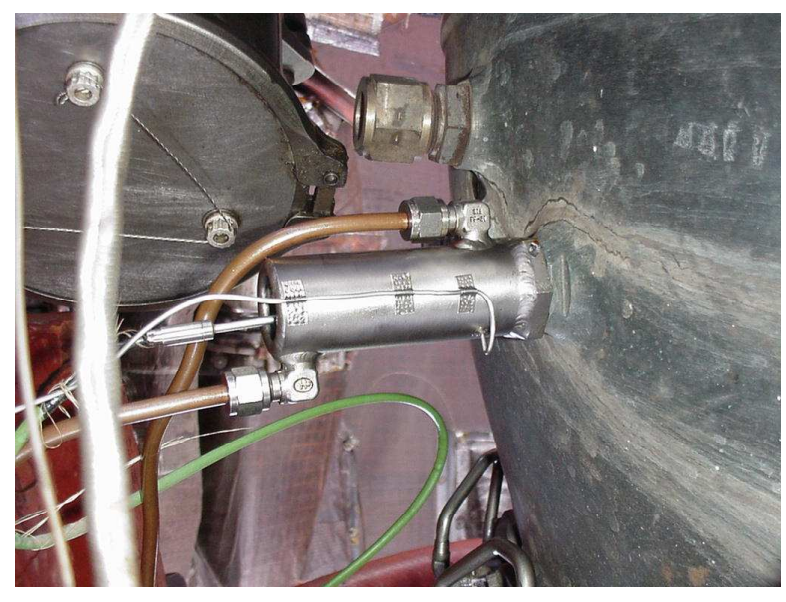

Figure 2. Kulite hardware mounted on PW4098 turbofan engine at Pratt \& Whitney test stand C11, West Palm Beach Florida for EVNRC Phase 2 tests.

In the analysis of aircraft acoustic core noise sources presented herein the measured signal is treated as a mixture of random data from stationary processes composed of time series data from "coherent" random processes, "incoherent" random processes and tones which are due to periodic/almost periodic deterministic processes. More generally, this is a situation with a time series having a mixed spectrum that is composed of contiuous and discrete parts. Many approaches to decompose this type of spectra into components have been tried. Early attempts are reviewed by Priestley. ${ }^{1}$ More recent attempts use solve the problem by estimating parameters of an autoregressive (AR) process or some other process ( Sengupta and Kay, ${ }^{2}$ Kay and Nagesha ${ }^{3,4}$ Ciuciu, Idier, and Giovannelli ${ }^{5}$ ). The aligned and unaligned coherence method provide means 
to separate this mixture so that noise components can be related to turbofan engine sources without a parameter estimation procedure.

Table 1. Spectral Estimate Parameters

\begin{tabular}{|l|l|}
\hline Parameter & value \\
\hline \hline Segment/block length i.e. Data points per segment/block, $N P$ & 4096 \\
Sample rate, $r$, samples/second & 48,000 \\
Segment length, $T_{d}=N P / r$, seconds & 0.08533 \\
Sampling interval, $\Delta t=1 / r$, seconds & $2.0833 \times 10^{-5}$ \\
Frequency step, $\Delta f=1 / T_{d}, \mathrm{~Hz}$ & 11.718 \\
Upper frequency limit, $f_{c}=1 / 2 \Delta t=r / 2, \mathrm{~Hz}$ & 24000 \\
number of frequencies, $L y=f_{c} / \Delta f=N P / 2$ & 2048 \\
Time delay, $\tau=6323 / 48000$, seconds & 0.1317 \\
number of independent segments/blocks & 234 \\
overlap & 0.50 \\
Sample length,sec. & 20 \\
\hline
\end{tabular}

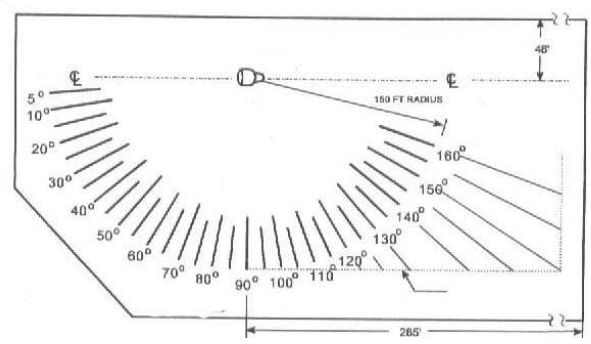

Figure 3. Acoustic arena and microphone array at Pratt \& Whitney test stand C11, West Palm Beach Florida for EVNRC Phase 2 tests. tion noise and create a one parameter measure of its presence or absence. It was shown that correlated combustion noise is only a small part of the total noise and that the plane wave combustion mode is appar- 
ent at low engine speeds. The aligned and unaligned coherence method used in this work is discussed herein. Results are illustrated with examples from studies made of the Pratt and Whitney P\&W 4098 turbofan engine data.

A review of previous work, the formulation of the three signal technique, and the formulation of the coherent power method are discussed in the appendix.

In the next section the experiment is discussed then the new diagnostic procedure is discussed.

\section{Experiment}

To demonstrate the usefulness of the proposed aligned and unaligned coherence method, Kulite pressure measurements made in a Pratt \& Whitney PW4098 combustor will be used. The measurements were made in a study of aircraft turbofan engine core noise conducted as part of the NASA Engine Validation of Noise Reduction Concepts (EVNRC) Program. One Kulite was at 127 degrees and the other was at 337 degrees. Kulite angles are measured clockwise from top dead center viewed from the rear or exhaust section of the engine.

The spectral estimate parameters are shown in table 1 on the preceding page The signal processing procedure used is discussed in the next section.

The test stand is shown in Figure 1 on page 3. The Kulites inside water cooled jackets mounted in the combustor are shown in Figure 2 on page 3. The angular placement of the far field microphones on a 150 foot radius is shown in Figure 3 on the page before. This analysis uses the microphones at $100^{\circ}, 110^{\circ}$, $120^{\circ}$ and $130^{\circ}$.

\section{Core Noise Study System Concepts}

In this paper signal I discuss signal analysis calculations which are well defined for deterministic signals. However, they are only defined for a limited classes of random signals. To simplify the discussion I will assume the random signals under discussion are from a 0 mean Gaussian stocahstic process. I will assume the process is ergodic so that the time-averaged statistics are the same as the ensemble-averaged statistics. I will assume the process is strict-sense stationary so that the parameters of the probability model of the process are time invariant. A discussion of a larger class of stochastic processes is given by Vaseghi ${ }^{9}$ (Chapter 3), Grimmett and Stirzaker, ${ }^{10}$ and Parzen. ${ }^{11}$ The signal analysis calculations discussed herein are calculations of the auto-spectrum and cross-spectrum. A discussion of various methods of calculating spectra are discussed by Vaseghi ${ }^{9}$ (Chapter 9) and Stearns and Hush. ${ }^{12}$

The procedure discussed herein involes the following items

- The estimated magnitude squared coherence (MSC) function, $\hat{\gamma}_{x y}^{2}(f)$. First, the MSC function will be discussed. Then, the estimation procedure will be discussed.

The magnitude squared coherence (MSC) function, $\gamma_{x y}^{2}(f)$ calculated using the complex cross-spectral density, $G_{x y}(f)$, and two auto-spectral densities at frequency f $G_{x x}(f)$ and $G_{y y}(f)$ is defined by:

$$
\gamma_{x y}^{2}(f)=\frac{\left|G_{x y}(f)\right|^{2}}{G_{x x}(f) G_{y y}(f)}
$$

The MSC function is discussed by Bendat and Piersol, ${ }^{13-15}$ Carter, ${ }^{16}$ Halliday ${ }^{17}$ et. al. and Jenkins and Watts ${ }^{18}$ where it is called the squared coherency.

The MSC function estimation procedure used herein are based on signal processing algorithms developed by Stearns and David. ${ }^{19}$ They refer to their spectral-estimation code as using a periodgram averaging method. The algorithm is also referred to a being based on Welch's method (Welch, ${ }^{20} \mathrm{McCellan}{ }^{21}$ ) and as a weighted overlapped segment averaging (WOSA) method (Carter Knapp and Nuttall, ${ }^{22}$ Carter and Nuttall ${ }^{23,24}$,Nuttall and Carter ${ }^{25}$ ). The term "WOSA" will be used in a generic sense herein rather than refer to a spcific code implementation. In the WOSA method, the finite data records of the two signals $x(t)$ and $y(t)$ are divided into $n_{s}$ (possibly) overlapping segments of length $T_{d}$; each segment is multiplied by a smooth weighting window, $w(\tau)$, the cross-spectra density, and the auto-spectal densities are computed using a fast Fourier transform for each segment, and then the averaged cross-spectra density and the auto-spectral densities are obtained from the $n_{s}$ segment. The estimated MSC, $\hat{\gamma}_{x y}^{2}(f)$ 
is the estimated cross-spectral density normalized by the estimated auto-spectral densities. The MSC and its estimate are bounded by zero and unity.

The estimated MSC function calculated using $n_{d}$ weighted Fourier transforms $\left.X \hat{(f}\right)_{i}$ and $Y(f)_{i}$ based on a segment record length $T_{d}$ is defined by

$$
\begin{aligned}
w(\tau) & =\left(1-\frac{|\tau|}{T_{d}}\right) \\
\hat{x}(\tau) & =w(\tau) x(\tau) \\
\hat{y}(\tau) & =w(\tau) y(\tau) \\
\hat{X}_{i}(f) & =\int_{0}^{T_{d}(i)} \hat{x}(\tau) \exp (-j 2 \pi f \tau) d \tau \\
\hat{Y}_{i}(f) & =\int_{0}^{T_{d}(i)} \hat{y}(\tau) \exp (-j 2 \pi f \tau) d \tau \\
\hat{\gamma}_{x y}^{2}(f) & =\frac{\left|\sum_{i=1}^{n_{s}} \hat{X}_{i}(f) \hat{Y}_{i}^{*}(f)\right|^{2}}{\sum_{i=1}^{n_{s}} \hat{X}_{i}(f) \hat{X}_{i}^{*}(f) \sum_{i=1}^{n_{s}} \hat{Y}_{i}(f) \hat{Y}_{i}^{*}(f)}
\end{aligned}
$$

Here, ${ }^{*}$ denotes the complex conjugate. In the procedure, $\hat{X}_{i}(f)$ and $\hat{Y}_{i}(f)$ are the fast Fourier transform of the ith weighted segment of the process $x(t)$ and $y(t)$.

The WOSA procedure is advocated by Carter and Nuttall ${ }^{23-25}$ since it gives stability and minimizes the impact of window sidelobes. Expressions for the mean and variance of WOSA spectral estimators are developed by Percival and Walden. ${ }^{26}$ Carter, Knapp and Nuttall ${ }^{22}$ empirically investigated the effect of overlap, as opposed to no overlap on coherence estimation using the WOSA method. Results show a decrease in bias and variance to the estimator with increasing overlap and it is suggested that 50-percent overlap is highly desirable when cosine (Hanning) weighting is used. Early implementations of the WOSA method are described by Welch, ${ }^{20}$ Bingham, Godfrey, and Tukey, ${ }^{27}$ and Hinich and Clay. ${ }^{28}$ An extension of the WOSA method is discussed by Nuttall and Carter. ${ }^{29}$ A survey of many power spectra estimation procedures starting with the periodogram is given by Brillinger ${ }^{30}$ ( Chapter 5 ). A review and comparison of many spectrum analysis techniques is given by Kay and Marple. ${ }^{31}$

To test, use, and validate the WOSA method certain assumptions are made about the time series and the procedure (Scannell and Carter, ${ }^{32,33}$ Nuttall and Carter, ${ }^{25}$ Carter, ${ }^{16}$ Carter, ${ }^{34}$ Kay and Marple. ${ }^{31}$ Some of the assumptions that characterize the method are viewed as limitations by Kay and Marple. ${ }^{31}$

1. In testing the method one uses time sequences which are from stochastic processes and are assumed to be 0 mean, jointly stationary Gaussian stochastic processes. This assumptions are made to make the mathematical investigation of stability tractable.

2. the data segments are independent.

3. the data segments are multiplied by a smooth weighting window to reduce sidelobe leakage

4. each data segment is sufficiently long to ensure adequate spectral frequency resolution and reduce the bias.

5. the number of the data segments, $n_{s}$, is sufficient to make the estimator achieve reliable statistical performance or one can state this as are requirement that large observation-time, $T_{T o t a l}$, resolutionbandwidth, $B e$ products are needed. This is due to the fact that for zero overlap $B e T_{\text {Total }}=n_{s}$ where $B e=\Delta f=1 / T_{d}$.

These assumptions have been tested to see how sensitive the WOSA method is to them $\left(\right.$ Carter $\left.^{34,35}\right)$. Carter $^{34}$ states the results are relatively insensitive to the Gaussian assumption. Consequently, these assumptions do not limit from to applying the WOSA method to turbofan noise where the measured process has deterministic components embedded in random noise. For example, the spectral density estimated is directly proportional to the power for a sinusoidal time process.

Two additional characteristics of the MSC estimation process is its sensitivity to the alignment or translation of the time series (Carter and Knapp ${ }^{35}$ and the inability to observe tones mixed with 
broadband noise in the spectrum. These points will be discussed later. One of the objective of this paper is to point out how deliberate mis-alignment of the time series can enable one to observe tones in the spectrum.

- The spectrum based on the three signal magnitude coherence function (Appendix B on page 33 )

$$
G_{u_{1} u_{1}}(f)=G_{y_{1} y_{1}}(f) \frac{\left|\gamma_{y_{1} y_{2}}(f)\right|\left|\gamma_{y_{1} y_{3}}(f)\right|}{\left|\gamma_{y_{2} y_{3}}(f)\right|}
$$

- The coherent output power function ( Appendix C on page 35 )

$$
W_{s_{F} s_{F}}(f)=\gamma_{x y}^{2}(f) G_{y y}(f)
$$

\section{Traditional Viewpoint}

First the traditional view on measuring coherence will be presented to pinpoint the "blind spots". A good place to start is a statement about the proper analysis procedures to obtain a coherence measurement as stated by Piersol $^{36}$ :

1. The instrumentation is calibrated to eliminate relative phase errors in the measured signals.

2. The received signals are aligned as required to eliminate time delay bias errors in the coherence calculation.

3. The number of averages $n_{d}$ is sufficiently large to suppress small sample bias errors in the coherence calculations.

\section{Relationship or Randomness}

At this point it must be mentioned that the problem of interest concerns situations where the measured coherence spectrum has an orderly structure but most of the coherence values are less than 0.1 . A measurement of the coherence of two independent random time series might also have a spectrum where most of the values are less than 0.1. Consequently, the paper discusses a method to decide if a given small coherence represents a relationship or randomness. The coherence between the Kulite sensors in the combustor and the far field microphones is small since the P\&W4098 combustor was designed to reduce correlated noise. It has many fuel nozzles each of which acts as an independent noise source. Since the coherence of interest are small and positive in this paper they are plotted on a logarithmic scale rather than a linear scale. The tools used to answer the question are statistical theory, simulation and the aligned and unaligned coherence technique.

\section{Statistical Estimates of Coherence}

\section{A. Bias, Variance, and Confidence Interval}

It is common to evaluate the significance of the measured magnitude squared coherence (MSC) function of the stochastic processes ( assumed to be 0 mean, jointly stationary Gaussian stochastic processes) by computing statistical estimates of the bias, $B$, variance, $V$, and confidence interval, $E_{I}$. Definitions for these quantities are given in statistical theory references such as Bronwlee ${ }^{37}$ and examples are usually given for simple distribution functions. The statistics of the MSC function and its distribution function are not simple. They are akin to those found in the study of multivariate statistical analysis or linear statistical models which involve Wishart probability density distributions. (Anderson, ${ }^{38}$ Graybill, ${ }^{39}$ Cramer ${ }^{40}$ ). However, statistical theories involving the MSC are even more complicated since use is made of a complex Wishart distribution (Goodman ${ }^{41}$ ). In obtaining estimates of the bias, $B$, variance, $V$, and confidence interval, $E_{I}$, of $|\hat{\gamma}|^{2}$ one uses the conditional probability of estimated of the MSC, $|\hat{\gamma}|^{2}$, given the true MSC, $|\gamma|^{2}$ and the number of independent segments/blocks, $n_{s}$, used in the spectral estimation procedure. This is expressed as $p\left(\left.|\hat{\gamma}|^{2}\left|n_{s},\right| \hat{\gamma}\right|^{2}\right)$ and will be referred to herein as a density function. Consequently, 


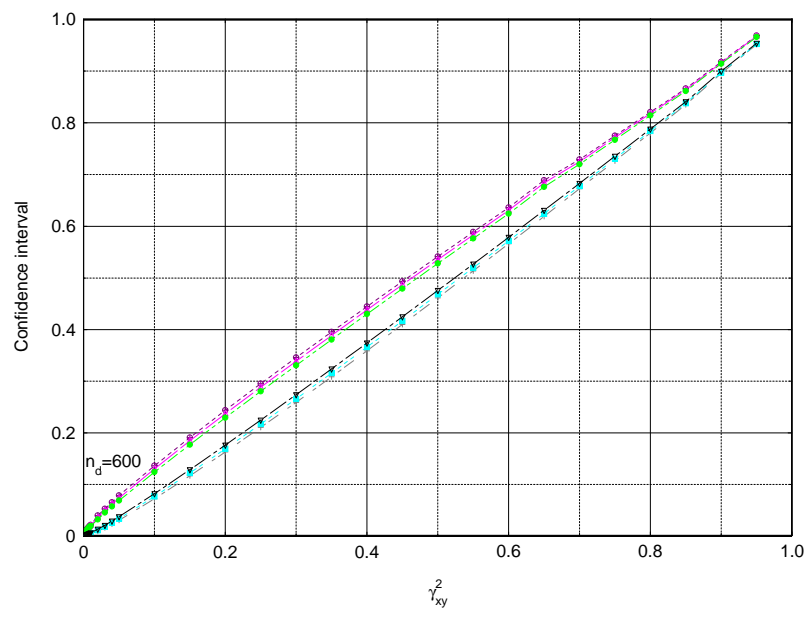

(a) Linear scale.

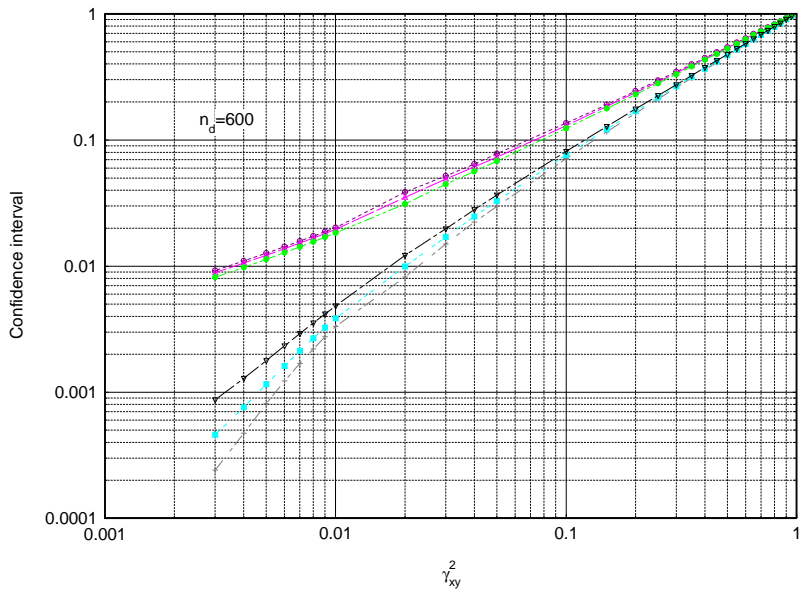

(b) Logorithmic scale.

Figure 4. Confidence interval for coherence function $n_{d}=600$.

$$
\begin{aligned}
B & =E\left[\left.|\hat{\gamma}|^{2}\left|n_{s},\right| \gamma\right|^{2}\right]-|\gamma|^{2} \\
V & =E\left[\left.|\hat{\gamma}|^{4}\left|n_{s},\right| \gamma\right|^{2}\right]-E^{2}\left[\left.|\hat{\gamma}|^{2}\left|n_{s},\right| \gamma\right|^{2}\right]
\end{aligned}
$$

The confidence interval $E_{I}$ can be calculated using the "equal tail areas" interval approch. We need to find $a$ and $b, a \leq|\hat{\gamma}|^{2} \leq b$ so $\alpha=.05$ to have the 95 percent confidence interval for $|\hat{\gamma}|^{2}$ using the relationship

$$
\int_{0}^{a} p\left(\left.|\hat{\gamma}|^{2}\left|n_{s},\right| \gamma\right|^{2}\right) d|\hat{\gamma}|^{2}=\int_{b}^{1} p\left(\left.|\hat{\gamma}|^{2}\left|n_{s},\right| \gamma\right|^{2}\right) d|\hat{\gamma}|^{2}=\frac{\alpha}{2}
$$

Assuming the Gaussian random processes have been divided into $n_{s}$ independent segments an analytical expression for the conditional probability density function of $|\hat{\gamma}|$ when $|\gamma|^{2}$ and $n_{s}$ are known, $p\left(\left.|\hat{\gamma}|\left|n_{s},\right| \gamma\right|^{2}\right)$, was first given by Goodman. ${ }^{41}$ It is also given by Hannan ${ }^{42}$ (page 253) for slightly less restrictive conditions that include the properties of Gaussian stochastic processes. The form given can written in terms of a hypergeometric function using Eq. 15.1.1 of Oberhettinger. ${ }^{43}$ Hannan $^{42}$ points out that the statistics do not hold at the zeroth and folding frequencies ( The first and last points in the spectrum). This conditional probability density function for $|\hat{\gamma}|$ must be converted to one for $|\hat{\gamma}|^{2}$ using the standard transformation of probability functions (Carter, ${ }^{22}$ Brownlee $^{37}$ ).

$$
\begin{aligned}
g & =|\hat{\gamma}|^{2} \\
y & =|\hat{\gamma}| \\
p\left(\left.|\hat{\gamma}|^{2}\left|n_{s},\right| \gamma\right|^{2}\right) & =\frac{p\left(\left.|\hat{\gamma}|\left|n_{s},\right| \gamma\right|^{2}\right)}{\frac{d g}{d y}}=\frac{1}{2|\hat{\gamma}|} p\left(\left.|\hat{\gamma}|\left|n_{s},\right| \gamma\right|^{2}\right) \\
& =\left(n_{s}-1\right)\left(1-|\gamma|^{2}\right)^{n_{s}}\left(1-|\hat{\gamma}|^{2}\right)^{n_{s}-2}{ }_{2} F_{1}\left(n_{s}, n_{s} ; 1 ;|\gamma|^{2}|\hat{\gamma}|^{2}\right) \quad 0 \leq|\gamma|^{2}|\hat{\gamma}|^{2}<1
\end{aligned}
$$

where ${ }_{2} F_{1}$ is the hypergeometric function (Eq. 15.1.1 of Oberhettinger ${ }^{43}$ )

This equation for $p\left(\left.|\hat{\gamma}|^{2}\left|n_{s},\right| \gamma\right|^{2}\right)$ is also given by Brillinger ${ }^{30}$ (Eq. 8.2.55) where we take $r=1$ in his equation Eq. 8.2.55.

Various forms of this probability density and the cumulative distribution function are tabulated by Carter. ${ }^{16,22}$ In addition, statistical estimates of coherence bias and coherence variance based on Eq. 8 are discussed by Carter. ${ }^{16,22}$ 
A significant limitation to the application of the MSC estimate is that for coherence values smaller than some fairly large value (like 0.1) and for large sample sizes the confidence interval is not available. For values greater than some value (like 0.1) the confidence interval is only given approximately ( Bendat and Piersol, ${ }^{13-15}$ Brillinger, ${ }^{30}$ Carter ${ }^{16,22}$ ) A procedure calculating the exact confidence interval is discussed by Wang et al. ${ }^{44,45}$ Consequently, if one calculates the upper and lower confidence interval one can determine the significance of the estimation and the significance of the differences of MSC values at different frequencies. Confidence limits are shown in Figure 4 on the preceding page for $n_{d}=600$. The confidence limits are shown on linear plot as they are usually shown (Scannell ${ }^{33}$ ) in Fig. 4a. The confidence limits are plotted in a more realistic manner in Fig. 4b on a logarithmic plot which demonstrates that for a given value of $n_{s}$ the confidence interval becomes larger as the value of $|\gamma|^{2}$ is reduced.

\section{B. Coherence of independent processes}

In considering the MSC of two stationary Gaussian random processes it is often stated that the MSC will be zero when the processes are independent and do not have a linear time-invariant relationship. However, the estimated MSC is never zero but has some numerical value. The only statement that can be made is that if the true MSC value is zero, $|\gamma|=0$, the expected value is in a certain confidence interval with a certain probability. The threshold value of this confidence interval will be called $E_{I}=\hat{\gamma}_{x_{n} y_{n}}^{2}$.

The conditional probability of the estimated value of the MSC, $|\hat{\gamma}|^{2}$, given the true MSC is zero, $|\gamma|^{2}=0$ and the number of independent segments/blocks, $n_{s}$, can be calculated from Eq. 8 as

$$
p\left(\left.|\hat{\gamma}|^{2}\left|n_{s},\right| \gamma\right|^{2}=0\right)=\left(n_{s}-1\right)\left(1-|\hat{\gamma}|^{2}\right)^{n_{s}-2}
$$

This equation is given by Brillinger ${ }^{30}$ as Eq. 8.2.55.

Then the probability, $P_{I}$ that $|\hat{\gamma}|^{2}$ is in the interval $0<|\hat{\gamma}|^{2}<E_{I}$

$$
\begin{aligned}
\hat{C} & =|\hat{\gamma}|^{2} \\
P_{I} & =\int_{0}^{E_{I}} p\left(\left.|\hat{\gamma}|^{2}\left|n_{s},\right| \gamma\right|^{2}=0\right) d|\hat{\gamma}|^{2} \\
& =\int_{0}^{E_{I}}\left(n_{s}-1\right)(1-\hat{C})^{n_{s}-2} d \hat{C} \\
& =-\left.(1-\hat{C})^{n_{s}-1}\right|_{0} ^{E_{I}} \\
& =-\left(1-E_{I}\right)^{n_{s}-1}+1
\end{aligned}
$$

Thus the cumulative distribution derived using he probability density function of the coherence derived by Goodman $^{41}$ has been used to determine a $P_{I}$-percent confidence interval if the true MSC is zero. This is given by

$$
\hat{\gamma}_{x_{n} y_{n}}^{2}=E_{I}=1-\left(1-P_{I}\right)^{1 /\left(n_{s}-1\right)}
$$

where $n_{s}$ is the number of independent segments used in the spectrum calculations and the interval is between 0 and $\gamma_{x_{n} y_{n}}^{2}$. This relationship is tabulated in Table 3 for probability values , $P_{I}$, of 80, 90, 95, and 99 percent. and is shown in figure 6 .

This statistical test for independence using Eq. (11) is discussed by Carter ${ }^{16,34}$ who proposes using $\gamma_{x_{n} y_{n}}^{2}$ as a threshold value below which coherence estimtes are not presented to a human decision maker looking at an extensive volume of data collected for brain wave analysis or sonar or radar signal analysis.

This equation is also given by Halliday ${ }^{17}$ et. al. page 247 and Brillinger ${ }^{30}$ page 317 .

Halliday suggests one should plot the value of $\gamma_{x_{n} y_{n}}^{2}$ calculated from Eq. (11) i.e. under the hypothesis of independence on each coherence plot. He states that estimated values of coherence lying below this line can be taken as evidence to indicate that the coherence is zero at that frequency. This opinion is shared by Nuttall $^{46}$ who thinks this is the standard result for a true coherence of zero. However, the author thinks it may indicate that not enough segments/blocks have been used to determine the coherence. One can state 
with more confidence that values above the $\gamma_{x_{n} y_{n}}^{2}$ line are not from two independent stationary random Gaussian series. Note that this statement is valid independent of the magnitude of $\gamma_{x_{n} y_{n}}^{2}$.

We have

It is also possible to calculate the bias and variance of $|\hat{\gamma}|^{2}$ when $|\gamma|^{2}=0$ using Eqs (5) (6) and (9).

$$
\begin{aligned}
\hat{C} & =|\hat{\gamma}|^{2} \\
B & =E\left[\left.|\hat{\gamma}|^{2}\left|n_{s},\right| \gamma\right|^{2}=0\right] \\
& =\int_{0}^{1} \hat{C}\left(n_{s}-1\right)(1-\hat{C})^{n_{s}-2} d \hat{C}=1 / n_{s} \\
V & =E\left[\left.|\hat{\gamma}|^{4}\left|n_{s},\right| \gamma\right|^{2}\right]-E^{2}\left[\left.|\hat{\gamma}|^{2}\left|n_{s},\right| \gamma\right|^{2}\right] \\
& =\int_{0}^{1} \hat{C}^{2}\left(n_{s}-1\right)(1-\hat{C})^{n_{s}-2} d \hat{C}-\frac{1}{n_{s}^{2}} \\
& =\frac{2}{n_{s}\left(n_{s}+1\right)}-\frac{1}{n_{s}^{2}}=\frac{n_{s}-1}{n_{s}^{2}\left(n_{s}+1\right)}
\end{aligned}
$$

Note that for $|\gamma|^{2}=0$ the bias, B, is the expected or mean value of $|\hat{\gamma}|^{2}$. and that for a large number of segments/blocks, $n_{s}$, these results agrees with those given by Carter. ${ }^{16,22}$

A reference to this result is made byTukey ${ }^{47}$ page 32 who states

In dealing with the real-valued measure of coherence, we must recall that wholly incoherent time series will show an average coherence of $\frac{2}{m}$, where the spectrum estimates are worth $\mathrm{m}$ degrees of freedom each.

The degrees of freedom, $m$, are defined as $m=2 B_{e} T_{\text {Total }}=2 n_{s}$. Consequently, Tukey states that

$$
E\left[\left.\left|\hat{\gamma}_{x_{n} y_{n}}\right|^{2}(f)\left|n_{s},\right| \gamma\right|^{2}=0\right]=\frac{1}{n_{s}}
$$

The fact that Tukey knew this is not surprising since he was the Ph.D thesis advisor of Goodman.

\section{Coherence and Simulation Methods}

Many assessments of the validity of the statistical estimates of coherence have been made using simulations. The probability density function of the coherence derived by Goodman ${ }^{41}$ was derived for Gaussian data. Foster and Guinzy ${ }^{48}$ tested this distribution by means of Monte Carlo experiments for validity and robustness (insensitivity to the Gaussian assumption) and it passed the tests. Coherence function bias and confidence intervals were studied using Monte Carlo methods by Benignus. ${ }^{49}$

The probability density function of the coherence derived by Goodman ${ }^{41}$ does not take into account the use of overlapping data. Empirical simulation results by Carter, Knapp and Nuttall ${ }^{16,22}$ show a decrease in bias and variance of the estimator with increasing overlap and suggest a 50-percent overlap as being highly desirable.

Furthermore, since the probability density function of the coherence derived by Goodman ${ }^{41}$ is independent of the computer code time series analysis implementation comparing bias and variance simulation results can provide some confidence in the analysis procedure. Simulations were done for a range of target coherence values and a range of independent segments/blocks using

$$
\begin{aligned}
& x_{n}(t)=n_{3}+\alpha n_{1} \\
& y_{n}(t)=n_{3}+\alpha n_{2} \\
& z_{n}(t)=n_{3}
\end{aligned}
$$

where $n_{1}, n_{2}$, and $n_{3}$ are independent Gaussian random time series.

The time series are selected so that

$$
\begin{aligned}
& G_{n_{3} n_{3}}=G_{n_{1} n_{1}}=G_{n_{2} n_{2}}=G_{s s} \\
& G_{n_{1} n_{2}}=G_{n_{3} n_{1}}=G_{n_{3} n_{2}}=0
\end{aligned}
$$




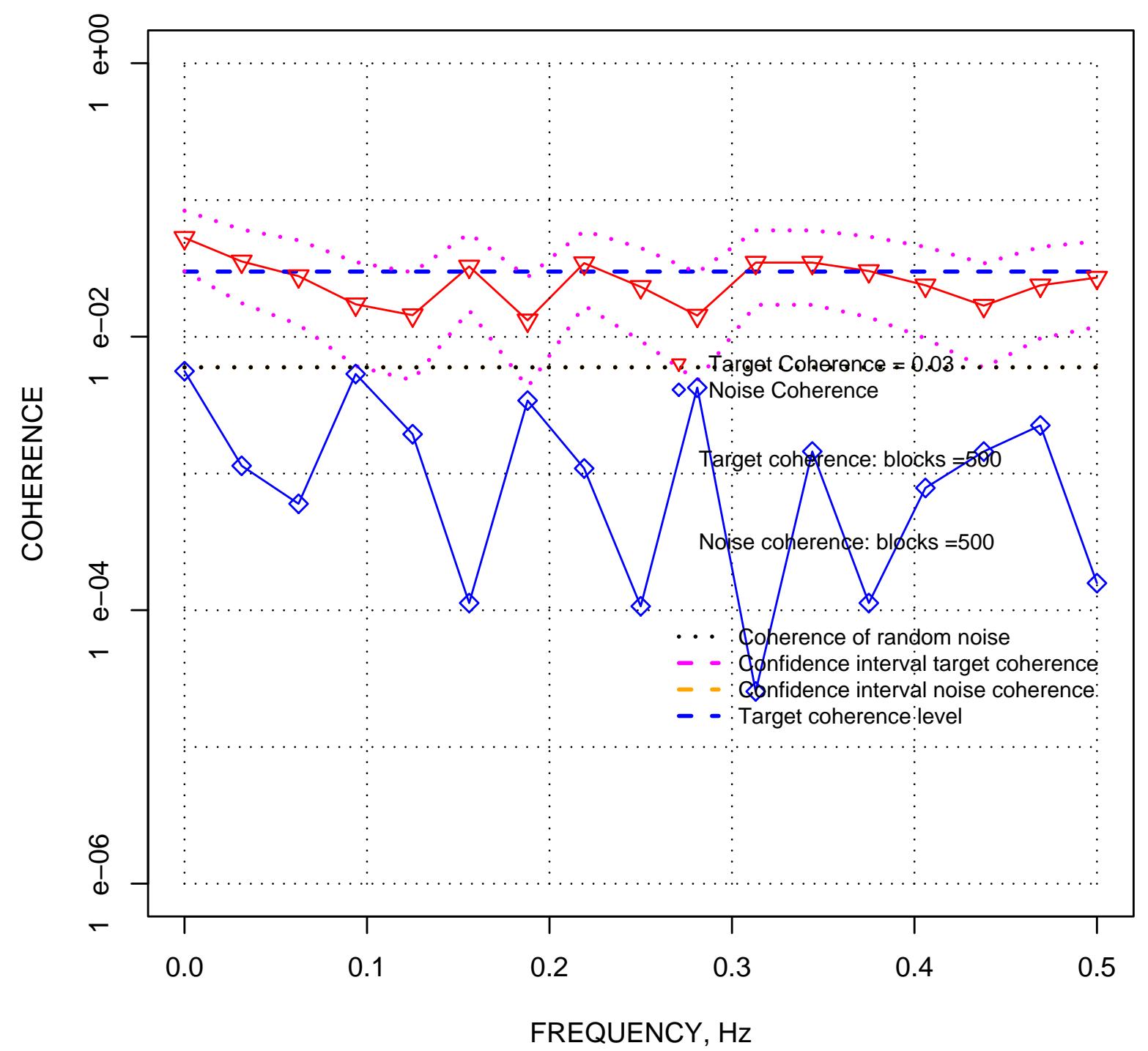

Figure 5. Simulation using target coherence $\gamma_{x y}^{2}=0.03$. Plot shows measaured coherence and noise coherence for $n_{d}=500 \gamma_{x_{n} y_{n}}^{2}(f, P=0.95)=0.005985$ and the confidence interval. 
Taking the Fourier transform of $x_{n}$ yields

$$
X_{x_{n}}(f)=X_{n_{3}}(f)+\alpha X_{n_{1}}(f)
$$

The spectrum $G_{x_{n} x_{n}}$ is then

$$
\begin{aligned}
G_{x_{n} x_{n}} & =X_{x_{n}}(f) X_{x_{n}}^{*}(f)=\left(X_{n_{3}}(f)+\alpha X_{n_{1}}(f)\right)\left(X_{n_{3}}(f)+\alpha X_{\left.\left(n_{1}\right)(f)\right)^{*}}\right. \\
& =G_{n_{3} n_{3}}+\alpha^{2} G_{n_{1} n_{1}}
\end{aligned}
$$

We then also have $G_{y_{n} y_{n}}=G_{n_{3} n_{3}}+\alpha^{2} G_{n_{2} n_{2}}$ and

$$
\begin{aligned}
G_{x_{n} x_{n}} & =G_{n_{3} n_{3}} \\
G_{x_{n} z_{n}} & =G_{n_{3} n_{3}} \\
G_{y_{n} z_{n}} & =G_{n_{3} n_{3}} \\
G_{z_{n} z_{n}} & =G_{n_{3} n_{3}}
\end{aligned}
$$

Then the target MSC is a function of $\alpha$ given by

$$
|\gamma|_{x_{n} y_{n}}^{2}=\frac{\left|G_{x_{n} y_{n}}\right|^{2}}{G_{x_{n} x_{n}} G_{y_{n} y_{n}}}=\frac{1}{\left(1+\alpha^{2}\right)^{2}}
$$

and

$$
|\gamma|_{x_{n} z_{n}}^{2}=|\gamma|_{y_{n} z_{n}}^{2}=\frac{1}{\left(1+\alpha^{2}\right)} .
$$

These expressions are independent of frequency. A similar approach is used to create target simulations by Carter, Knapp, and Nuttall ${ }^{22}$ Eq. (18) and Foster and Guinzy ${ }^{48}$ Eq. (13). Simulation results using a target coherence of $|\gamma|_{x y}^{2}=0.03$ are shown in Figure 5 on the page before. The coherence is low so a logarithmic coherence scale is used. The target coherence is shown by the dashed blue line. The plot shows the estimated or measured coherence, $|\hat{\gamma}|_{x_{n} y_{n}}^{2}(f)$, as red triangles and an estimated or measured noise coherence, $|\hat{\gamma}|_{n_{1} n_{2}}^{2}(f)$ as blue triangles for $n_{s}=500$. The upper and lower confidence interval is shown as pink and orange dashed lines. In addition, the horizontal line $\gamma_{x_{n} y_{n}}^{2}(f, P=0.95)=0.005985$ is shown using black dots. For this case the estimated noise coherence is less than $\gamma_{x_{n} y_{n}}^{2}(f, P=0.95)$ and the measured coherence is well above 0.00595. Consequently, the coherence while low is significant.

The results of a great many simulations are shown in figure 6 on the following page where average MSC values, $\bar{\gamma}_{x y}^{2}$ are presented as a function of the number of independent segments/blocks,$n_{s}$. Each simulation resembles the one shown in figure 5 on the page before and is designed for a particular target value. We use as a measure of convergence to a particular target value

$$
\bar{\gamma}_{x y}^{2}=\frac{1}{n} \sum_{i=0}^{n-1}|\hat{\gamma}|_{x y}^{2}\left(f_{i}\right)
$$

Simulations with a range of target MSC values calculated using Eq. (14) and (15) are shown. The colored symbols trace each target value. The target values are given in the legend. In addition, simulation results for the mean value when two independent simulated random Gaussian time histories are used to calculate $|\hat{\gamma}|_{n_{1} n_{2}}^{2}(f)$ are shown as black triangles and identified as $\gamma_{x y}^{2}=0$ in the legend.

The results show clearly that a coherent pair of signals will have a coherence that resembles the coherence of random noise if the number of segments/blocks, $n_{s}$, is too small. For example, for $n_{s}=100$ one would see an indication of $M S C=0.01$ but not of $M S C=0.0025$. Consequently, the value $n_{s}$ controls not only the bias and variance of the coherence but also its existence. Consequently, for a given measurement of coherence using a given value of $n_{s}$ one can say that a coherence may not be observed if there is a $P$-percent probability that it is less than $\hat{\gamma}_{x_{n} y_{n}}^{2}\left(f, P_{I}\right)=E_{I}=1-\left(1-P_{I}\right)^{1 /\left(n_{s}-1\right)}$ (Eq. 11$)$. This curve is identified as $y=1-0.05^{x}$ in the legend box. This curve is shown in the figure as a red line for a 95 percent confidence interval. In addition, the $\gamma_{x y}^{2}=1 / n_{s}$ line is shown as a diagonal black line identified as $y=x$ in the legend. This line corresponds to the expected or mean value of $|\bar{\gamma}|_{x y}^{2}$ for $|\gamma|_{x y}^{2}=0$ as given in Eq. (12). This curve 
also corresponds to the one Tukey ${ }^{47}$ suggested as discussed in the previous section. The estimated mean value of $|\hat{\gamma}|_{n_{1} n_{2}}^{2}(f)$ shown by the black triangles is close to this line However, The 95 percent confidence interval provides a better number to be larger than when one wishes to identify a small coherence value as being significant.

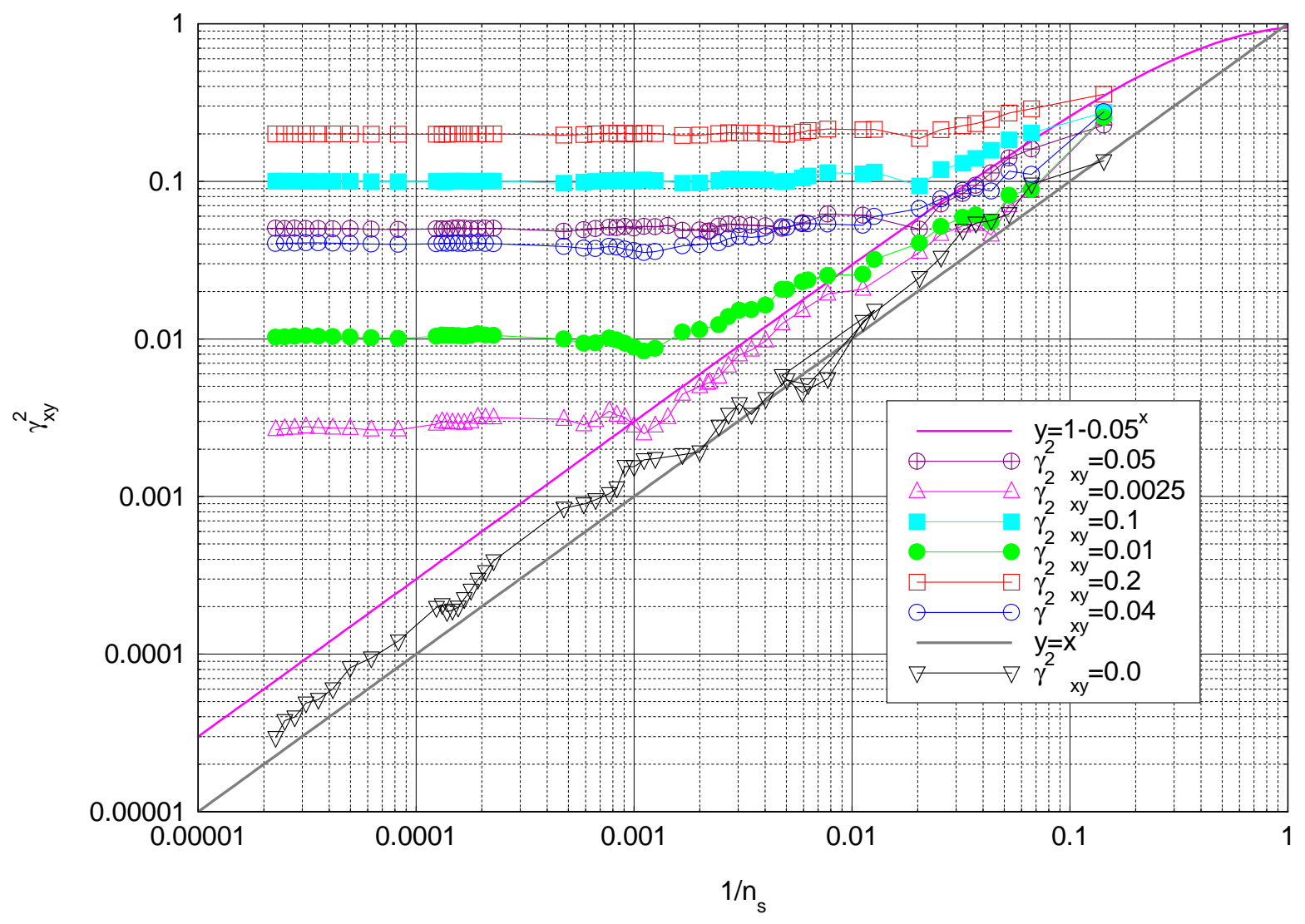

Figure 6. Simulation result summary.

\section{Aligned and Unaligned Coherence}

\section{A. Time delay bias error}

One explanation for the time delay bias error starts by considering two measurement points separated by a distance $\Delta x$. The transfer function between the two measurement points is $\exp (j k \Delta x)=\exp \left(j 2 \pi f \Delta x / c_{o}\right)$ where $k$ is the wave number, $f$ the frequency, and $c_{o}$ the speed of sound. The phase angle is $\theta=2 \pi f \Delta x / c_{o}$. For a situation where the cross-spectrum between the two points is calculated Tick ${ }^{50}$ writes:

Then for increasing $f$, the phase angle changes at an increasing rate. At some frequency the cross-spectrum will oscillate sufficiently rapidly that the expected value of the cross-spectrum estimate will be zero.

In regards to this type of time delay bias error Tukey ${ }^{47}$ wrote

If we fail to fix "zero lag" between the two series appropriately, we can almost wholly lose sight of the actual cross-spectrum (by averaging over narrow frequency bands within which the phase relation revolves one or more complex revolutions. 
The second explanation puts these vague words about expected value and averaging in a more mathematical form. The account given should serve as representation of the origin of the time delay bias. The following discussion follows along the same line of reasoning used by Seybert and Hamilton ${ }^{51}$ and by Carter $^{52}$ but tries to relate the formulation to the average periodogram method of spectral estimation ( Stearns and Hush, ${ }^{12}$ Vaseghi $^{9}$ ).

The cross-spectral density $S_{x y}$ between two stationary random ergodic Gaussian processes $x(t)$ and $y(t)$ is estimated from a finite length time record, $T_{k}$ by

$$
S_{x y}(f, T, k)=\frac{1}{T} X(f, T, k) X^{*}(f, T, k)
$$

For a stochastic process the spectrum $S_{x y}$ is defined as

$$
S_{x y}=\lim _{\mathrm{T} \rightarrow \infty} E\left[S_{x y}(f, T, k)\right]
$$

where $E\left[S_{x y}(f, T, k)\right]$ means the expected value operation is done over the ensemble index $\mathrm{k}$ which is record length, $T_{k}$ index. However, to be more precise we are using an averaged periodogram procedure. The periodograms are averaged to produce the spectral estimate. To arrive at the final equation we define the average of the cross spectral densities.

$$
\bar{\phi}_{x y}(f, T)=\frac{1}{K} \sum_{i=1}^{k} S_{x y}(f, T, k)
$$

If the cross-spectral densities are independent then spectrum, $S_{x y}(f, T)$, is the expected value of the sum $\bar{\phi}_{x y}(f, T)$.

$$
S_{x y}(f)=E\left[\bar{\phi}_{x y}(f, T)\right]=\frac{1}{K} \sum_{i=1}^{K} E\left[S_{x y}(f, T, i)\right]=\frac{1}{K} \sum_{i=1}^{K} I_{x y}^{i}(f, T)
$$

where the average periodogram is defined as $I_{x y}^{k}(f, T)=E\left[S_{x y}(f, T, k)\right]$

It can be shown that the average periodogram is

$$
I_{x y}^{k}(f, T)=E\left[S_{x y}(f, T, k)\right]=\int_{-T_{k}}^{T_{k}}\left(1-\frac{|\tau|}{T}\right) R_{x y}(\tau) \exp (-j 2 \pi f \tau) d \tau
$$

where $R_{x y}(\tau)$ is the cross-correlation function between $x(t)$ a and $y(t)$.

This expression appears in Priestley ${ }^{1}$ on page 417 and is explained for discrete formulations on page 319. A derivation of Eq. (23) is given by Bendat and Piersol ${ }^{14}$ section 3.2.3. A similar derivation is given by Davenport and Root ${ }^{53}$ page 106 and Jenkins and Watts ${ }^{18}$ section 6.2.1. The derivation is sketched in Appendix D on page 36.

The expected values of the one sided cross-spectra and auto-spectra periodograms of $x(t)$ and $y(t)$ are

$$
\begin{aligned}
& J_{x y}^{k}(f, T)=E\left[G_{x y}(f, T, k)\right]=\int_{0}^{T_{k}} 2\left(1-\frac{|\tau|}{T}\right) R_{x y}(\tau) \exp (-j 2 \pi f \tau) d \tau \\
& J_{x x}^{k}(f, T)=E\left[G_{x x}(f, T, k)\right]=\int_{0}^{T_{k}} 2\left(1-\frac{|\tau|}{T}\right) R_{x x}(\tau) \exp (-j 2 \pi f \tau) d \tau \\
& J_{y y}^{k}(f, T)=E\left[G_{y y}(f, T, k)\right]=\int_{0}^{T_{k}} 2\left(1-\frac{|\tau|}{T}\right) R_{y y}(\tau) \exp (-j 2 \pi f \tau) d \tau
\end{aligned}
$$

The one sided spectra are then given by

$$
\begin{aligned}
G_{x y}(f) & =\frac{1}{K} \sum_{i=1}^{K} J_{x y}^{i}(f, T) \\
G_{x x}(f) & =\frac{1}{K} \sum_{i=1}^{K} J_{x x}^{i}(f, T)
\end{aligned}
$$




$$
G_{y y}(f)=\frac{1}{K} \sum_{i=1}^{K} J_{y y}^{i}(f, T)
$$

We now use these definitions to study the time delay bias problem using the white noise example found in Seybert and Hamilton. ${ }^{51}$ Seybert and Hamilton ${ }^{51}$ derived an equation for the time delay bias for this case provided one uses an average periodogram type calculation procedure to calculate MSC .

Let $x(t)$ and $y(t)$ be Gaussian, white noise stochastic processes. For a white noise the spectrum is constant at all frequencies. Thus the spectra are $G_{x y}=G_{x x}=G_{y y}=A$ and the $|\gamma|_{x y}^{2}=1$.

For this case the autocorrelation functions are $R_{x x}=R_{y y}=A \delta(\tau)$ (Bendat ${ }^{13}$ page 85) Since $y(t)$ is delayed

$$
\begin{aligned}
& y(t)=x\left(t-\tau_{o}\right) \\
& R_{x y}=A \delta\left(\tau-\tau_{o}\right)
\end{aligned}
$$

Substituting these relations into Eqs.(23) through (29) we have for the spectra.

$$
\begin{aligned}
G_{x y} & =J_{x y}^{k}(f, T)=A\left(1-\frac{\left|\tau_{o}\right|}{T}\right) \exp \left(-j 2 \pi f \tau_{o}\right) \\
G_{x x}=G_{y y}=J_{x x}^{k}(f, T) & =J_{y y}^{k}(f, T)=A
\end{aligned}
$$

Consequently, with a time delay, $\tau_{o}$ the estimated MSC for this case , $|\hat{\gamma}|_{x y}^{2}$ for this case is

$$
|\hat{\gamma}|_{x y}^{2}=\frac{\left|G_{x y}\right|^{2}}{G_{x x} G_{y y}}=\left(1-\frac{\left|\tau_{o}\right|}{T}\right)^{2}|\gamma|_{x y}^{2}
$$

This relationship has been used to explain time delay bias by Halvorsen and Bendat, ${ }^{54}$ Seybert and Hamilton, ${ }^{51}$ Bendat and Piersol ${ }^{15}$ in chapter 11.2 (page 271) and Carter. ${ }^{16,52}$ The importance of eliminating time delay bias and realigning time series by dsisplacing one of them with respect to the other has been mentioned in books by Bendat ${ }^{13}$ section 7.4.5, Jenkins and Watts ${ }^{18}$ page 399-400, Koopmans ${ }^{55}$ page 309, and Brillinger ${ }^{30}$ page 266 and in articles by Tukey ${ }^{47}$ p32 Tick $^{56}$ p 135 , Halvorsen and Bendat, ${ }^{54}$ Carter $^{16,22,52}$ Seybert and Hamilton, ${ }^{51}$ and Trethewey and Evensen. ${ }^{57}$

The importance of using the correct model for the spectrum analysis process will be illustrated using an analysis provided by Priestly ${ }^{1}$ on page 663 where an example is derived for linear regression with delay. This is a discrete parameter process problem and will be discussed using the appropriate discrete definitions (see Priestley ${ }^{1}$ section 4.8.3). The objective of the analysis was to show the invariance of coherency under linear transformations. However, this example will be used to show the importance of using the correct expression for $E\left[S_{x y}(f, T, K)\right]$ (Appendix D on page 36 ). The discussion starts on page 657 using expressions of the form

$$
h(\omega)=\frac{1}{2 \pi} \sum_{s=-\infty}^{\infty} R(s) \exp (-j s \omega)
$$

to define spectra as Fourier transforms of the respective auto and cross covariance functions, $R(s)$. This expression is also found as equation (4.8.34) on page 225. The next step is to let $X_{1, t}$ and $X_{2, t}$ satisfy a linear regression without time delay relationship of the form

$$
X_{1, t}=a X_{2, t}+\epsilon_{t}
$$

where $\epsilon_{t}$ is a white noise process uncorrelated with $X_{2, t}$. Here, we change the example slightly and assume that $X_{1, t}$ are $X_{2, t}$ also has a white noise spectrum so that we have expressions for auto and cross covariance functions. We then have

$$
\begin{aligned}
& R_{\epsilon \epsilon}(s)=b \delta(s) \\
& R_{12}(s)=a R_{22}(s)=a \delta(s)
\end{aligned}
$$




$$
\begin{aligned}
h_{11}(\omega) & =a^{2}+b^{2} \\
h_{22}(\omega) & =1 \\
h_{12}(\omega) & =a h_{22}(\omega)=a \\
\gamma_{12}(\omega) & =\frac{h_{12}}{\sqrt{h_{11}} \sqrt{h_{22}}} \\
& =\frac{a}{\sqrt{a^{2}+b^{2}}} \\
\left|\gamma_{12}(\omega)\right|^{2} & =\frac{\left|h_{12}\right|^{2}}{h_{11} h_{22}} \\
& =\frac{|a|^{2}}{a^{2}+b^{2}}
\end{aligned}
$$

where $h_{11}(\omega)$ and $h_{22}(\omega)$ are auto-spectra and $h_{12}(\omega)$ is the cross-spectra. The coherence is $\gamma_{12}(\omega)$ and the MSC is $\left|\gamma_{12}(\omega)\right|^{2}$.

We continue his example by next assuming that $X_{1, t}$ and $X_{2, t}$ satisfy a linear regression with a time delay of d time units. Then

$$
X_{1, t}=a X_{2, t-d}+\epsilon_{t}
$$

Then in this case

$$
R_{12}=a R_{22}(s-d)=a \delta(s-d)
$$

Then from his equation (9.1.13)

$$
\begin{aligned}
h_{21} & =\frac{1}{2 \pi} \sum_{s=-\infty}^{\infty} R_{21}(s) \exp (-j s \omega) \\
& =\frac{a}{2 \pi} \sum_{s=-\infty}^{\infty} a R_{22}(s-d) \exp (-j s \omega) \\
& =a \exp (-j \omega d)
\end{aligned}
$$

To summarize we have

$$
\begin{aligned}
R_{\epsilon \epsilon}(s) & =b \delta(s) \\
R_{12}(s) & =a R_{22}(s)=a \delta(s-d) \\
h_{11}(\omega) & =a^{2}+b^{2} \\
h_{22}(\omega) & =1 \\
h_{12}(\omega) & =a h_{22}(\omega)=a \exp (-j \omega d) \\
\gamma_{12}(\omega) & =\frac{h_{12}}{\sqrt{h_{11}} \sqrt{h_{22}}} \\
& =\frac{a \exp (-j \omega d)}{\sqrt{a^{2}+b^{2}}} \\
\left|\gamma_{12}(\omega)\right|^{2} & =\frac{\left|h_{12}\right|^{2}}{h_{11} h_{22}} \\
& =\frac{|a|^{2}}{a^{2}+b^{2}}
\end{aligned}
$$

Priestley then points out that the magnitude has not changed which shows an invariance of coherence under a linear transformationand. Next it is pointed out that the phase spectrum is a linear function of frequency which has a slope representing the magnitude of the delay. However, if for the spectra analysis equations used were the averaged periodogram equations discussed by Priestly ${ }^{1}$ in section 6.2 .3 for a continuous spectrum 
the results would not show the invariance of coherence under a linear transformation. We have using Eqs.. $(6.2 .54)$ and $(6.2 .65)$

$$
E[\hat{h}(\omega)]=\frac{1}{2 \pi} \sum_{s=-(N-1)}^{(N-1)}\left[1-\frac{|s|}{M}\right] R(s) \exp (-j s \omega)
$$

where $M<(N-1)$ is the number of points in a block. Since the spectra of interest is from a white noise source all blocks are identical and the block average is the same as the result for each block.

Then for the time delayed case where the delay is $d$ units of the sequence.

$$
\begin{aligned}
E[\hat{h}(\omega)] & =\frac{1}{2 \pi} \sum_{s=-(N-1)}^{(N-1)}\left[1-\frac{|s|}{M}\right] R_{21}(s) \exp (-j s \omega) \\
& =\frac{a}{2 \pi} \sum_{s=-(N-1)}^{(N-1)}\left[1-\frac{|s|}{M}\right] a R_{22}(s-d) \exp (-j s \omega) \\
& =a\left[1-\frac{|d|}{M}\right]^{2} \exp (-j \omega d)
\end{aligned}
$$

Consequently,

$$
\begin{aligned}
h_{11}(\omega) & =a^{2}+b^{2} \\
h_{22}(\omega) & =1 \\
h_{12}(\omega) & =a\left[1-\frac{|d|}{M}\right] \exp (-j \omega d) \\
\gamma_{12}(\omega, d) & =\frac{h_{12}}{\sqrt{h_{11}} \sqrt{h_{22}}} \\
& =\left[1-\frac{|d|}{M}\right] \frac{a \exp (-j \omega d)}{\sqrt{a^{2}+b^{2}}} \\
\left|\gamma_{12}(\omega, d)\right|^{2} & =\frac{\left|h_{12}\right|^{2}}{h_{11} h_{22}} \\
& =\left[1-\frac{|d|}{M}\right]^{2} \frac{|a|^{2}}{a^{2}+b^{2}} \\
& =\left[1-\frac{|d|}{M}\right]^{2}\left|\gamma_{12}(\omega)\right|^{2}
\end{aligned}
$$

Thus we have shown that when the spectrum calculation procedure is performed using the discrete form of $E\left[S_{x y}(f, T, K)\right]$ (Appendix D on page 36 ), the time delay bias appears again. The coherence is not invariant under a linear transformation when it is calculated using an averaged periodogram procedure. We find again, that if the time delay, $d$, exceeds the block size $M$ the coherence goes to zero. These points are not made by Priestley since in this example the analyzing procedure was one based on the original periodogram procedure instead of an averaged periodogram procedure. Priestly ${ }^{1}$ does discuss translating the time-axis of one process relative to the other so as to obtain the maximum cross-correlation at zero lag on page 712 .

Equation (23) is often considered as being equivalent to the Fourier transform of the true crosscorrelation function multiplied by a window function of the form

$$
w_{\tau}=1-\frac{\tau}{T}
$$

This is the Bartlett triangular window function. The convolution theorem show that

$$
I_{x y}^{k}(f, T)=E\left[S_{x y}(f, T, k)\right]=\int_{-\infty}^{\infty} W\left(f-f^{\prime}\right) P\left(f^{\prime}\right) d f^{\prime}
$$


where $W(f)$ is the Fourier transform of $w(\tau)$. The Fourier transform of the Bartlett triangular window is called the Fejer kernel, $F_{N}(f)\left(\right.$ Priestley $^{1}$ Section 6.2$)$ and

$$
F_{N}(f)=\int_{-T}^{T}\left(1-\frac{|\tau|}{T}\right) \exp (j 2 \pi f \tau) d \tau=T\left[\frac{\sin (\pi f T)}{(\pi f T)}\right]^{2}
$$

This function has a broad central peak and small side lobes which peak at odd multiples of $\frac{1}{2 T}$ that is at $\frac{n}{2 T}, \quad n= \pm 1, \pm 3, \pm 5 \ldots$.

From equation (35)

$$
I_{x y}^{k}(f, T)=E\left[S_{x y}(f, T, k)\right]=\int_{-\infty}^{\infty} F_{N}\left(f-f^{\prime}\right) P\left(f^{\prime}\right) d f^{\prime}
$$

This indicates that the Bartlett window results in a smoothing of the true spectrum but introduces a rippling effect. Accordingly, the MSC is "smeared out".

As $T \rightarrow \infty, F_{N}(f) \rightarrow \delta(f)$ ( the Dirac $\delta$-function)/. Consequently,

$$
I_{x y}^{k}(f, T \rightarrow \infty)=E\left[S_{x y}(f, T \rightarrow \infty, k)\right]=\int_{-\infty}^{\infty} \delta\left(f-f^{\prime}\right) P\left(f^{\prime}\right) d f^{\prime}=P(f)
$$

This suggests the importance of selecting a window function. One commonly used window, suggested by R. W. Hamming is

$$
w(\tau)=\left\{\begin{aligned}
0.54+0.46 \cos \left(\frac{\pi \tau}{T}\right) & : \text { for }|\tau|<T \\
0 & : \text { otherwise }
\end{aligned}\right.
$$

This window reduces the magnitude of the peak in the first side lobe of the spectral window relative to the magnitude of the peak in the main lobe. This transformation is made since windowing creates "leakage" in the spectral domain. This is the name for the transfer of energy in the main lobe of a spectral response function into the sidelobes obscuring and distorting other spectral responses that are present. Many window functions of interest are discussed by Priestly ${ }^{1}$ (section6.2.3). A good review of spectral analysis is given by Kay and Marple. ${ }^{31}$

Now the time-delay bias error given in equations (33) and (39) only applies to the Bartlett window. An investigation of the effect of the type of spectral analysis window used has been made by Trethewey and Evensen. ${ }^{57}$ They found the a MSC bias error depends on the window. In addition theoretically and experimentally they found that the MSC time delay the bias error was larger for a Hanning window for $\frac{|\tau|}{T}>0.2$ than for a Barlett window.

\section{B. Unaligned Coherence}

The method of aligned and unaligned coherence is based on the following two characteristics of the average periodogram process when it is used to calculate the MSC :

- When the time delay, $\tau_{1}$, exceeds the sample record length,$T_{d}$, the coherence of the random process, $\hat{\gamma}_{x y}^{2}(f)$, is the coherence of two independent random signals, $\hat{\gamma}_{x_{n} y_{n}}^{2}(f)$.

- If the signals contain tones using a time delay, $\tau_{1}$, greater than the sample record length,$T_{d}$, does not change the coherence of the tones.

In the next two sections these points will be discussed.

\section{Low coherence example}

To apply the aligned and unaligned coherence procedure, we first consider the measurement of a correlated Gaussian stationary noise source with time history, $s(t)$, in a situation where the measurement is contaminated with a Gaussian stationary random noise, $n$, where the random noise and the correlated noise are independent. As an example, this could be the measurement of presssure at two points in a turbofan combustor . Then 


$$
\begin{aligned}
& x_{1}(t)=s_{1}(t)+n_{1}(t) \\
& y_{2}(t)=s_{2}(t)+n_{2}(t)
\end{aligned}
$$

The auto-and cross spectra for this case are

$$
\begin{aligned}
G_{x_{1} y_{2}} & =G_{s_{1} s_{2}}(f)+N_{n_{1} n_{2}}(f) \\
G_{x_{1} x_{1}} & =G_{s_{1} s_{1}}(f)+N_{n_{1} n_{1}}(f) \\
G_{y_{2} y_{2}} & =G_{s_{2} s_{2}}(f)+N_{n_{2} n_{2}}(f)
\end{aligned}
$$

where $N(f)$ is used to designate the spectrum of the noise and $G(f)$ is used to designate the spectrum of the signal.

The estimated MSC is then

$$
\left|\hat{\gamma}_{x y}(f)\right|^{2}=\frac{\left|G_{s_{1} s_{2}}(f)+N_{n_{1} n_{2}}(f)\right|^{2}}{\left[G_{s_{1} s_{1}}(f)+N_{n_{1} n_{1}}(f)\right]\left[G_{s_{2} s_{2}}(f)+N_{n_{2} n_{2}}(f)\right]}
$$

The noise spectrum due to the uncorrelated random signals $n_{1}(t)$ and $n_{s}(t)$ is retained in this system equation since the magnitude of the cross spectrum of the signals much smaller than the auto-spectrum. The MSC under consideration looks that shown in Fig. 7.

The problem then is to find a way to show the measured low coherence is not due to noise. One method that is available is the method of surrogate data ( Faes. ${ }^{58}$ Theiler, ${ }^{59}$ Schreiber ${ }^{60}$ ). According to this method a set of surrogate series mimicking some properties of the original series but being otherwise uncoupled is generated. The threshold for zero coherence is then computed on the distribution of coherence estimates obtained from the surrogate series. For example, one of the procedures used by Faes to generate a surrogate series was to randomly permute in temporal order the segments/blocks of the original series so that any temporal structure was destroyed in the surrogate generation.

Another method to show the measured low coherence is not due to noise would be to compare the measured MSC with the 95 percent confidence interval for the same number of segments/blocks given by Eq. (11). A third method is to compare aligned and unaligned coherence function. Again, we start with the time series $x_{1}(t)$ and $y_{2}(t)$. However, we now delay the signal by an amount $D$ which is greater than the record length, T. As discussed in the previous section this decorrelates the signals. The signals can be treated as if they came from two independent random sources. The signals analyzed are now

$$
\begin{aligned}
x_{1}(t) & =s_{1}(t)+n_{1}(t) \\
y_{2}(t-D) & =s_{2}(t-D)+n_{2}(t-D)
\end{aligned}
$$

The auto-and cross spectra for this case are

$$
\begin{aligned}
G_{x_{1} y_{2}} & =N_{n n}(f) \\
G_{x_{1} x_{1}} & =G_{s_{1} s_{1}}(f)+N_{n_{1} n_{1}}(f) \\
G_{y_{2} y_{2}} & =G_{s_{2}(t-D) s_{2}(t-D)}(f)+N_{n_{2}(t-D) n_{2}(t-D)}(f)
\end{aligned}
$$

Note, that the cross-spectrum is now just the spectrum of two independent noise sources. The cross-spectrum of two signals when they are misaligned by a time greater than the block length is the spectrum of two independent noise sources when this is added to the cross spectrum of random noise the result is again a random noise spectrum.

The estimated MSC is then

$$
\left|\hat{\gamma}_{x y}(f, D \quad D>T)\right|^{2}=\frac{\left|N_{n n}(f)\right|^{2}}{\left[G_{s_{1} s_{1}}(f)+N_{n_{1} n_{1}}(f)\right]\left[G_{s_{2}(t-D) s_{2}(t-D)}(f)+N_{n_{2}(t-D) n_{2}(t-D)}(f)\right]}
$$


The signal auto-spectrum and the noise auto-spectrum are invariant with time shifting. Consequently,

$$
\begin{aligned}
N_{s_{2} s_{2}} & =N_{s_{2}(t-D) s_{2}(t-D)} \\
G_{s_{2} s_{2}} & =G_{s_{2}(t-D) s_{2}(t-D)}
\end{aligned}
$$

Thus

$$
\left|\hat{\gamma}_{x y}(f, D \quad D>T)\right|^{2}=\frac{\left|N_{n n}(f)\right|^{2}}{\left[G_{s_{1} s_{1}}(f)+N_{n_{1} n_{1}}(f)\right]\left[G_{s_{2} s_{2}}(f)+N_{n_{2} n_{2}}(f)\right]}
$$

Note that by obtaining the MSC of the unaligned signals we have established the level of coherence for two independent signals measured and processed with the same procedures and equipment as the aligned signals. All the results presented in section VII where the MSC was calculated using simulated independent Gaussian random time series now may be attributed to the unaligned MSC. In particular, the results shown in figure 6 on page 13 for $\left|\hat{\gamma}_{x_{n} x_{b}}\right|^{2}$ apply to the unaligned MSC.

Consequently, a comparison of the results immediately determines the validity of the low MSC measurement. An example, is shown in Fig. 7 and will be discussed in the results section of this paper. In addition, since the value of the aligned and unaligned MSC is derived by direct calculation using measured data one can obtain results for cases where the data blocks are overlapped instead of being independent. In addition, any problems due to windowing that change the effective number of segments/blocks, the averaging, or the energy leakage from the main lobe to side lobes is also accounted for.

All the findings provided in the previous section dealing with two independent random series applies to the unaligned and aligned time series if no tones are present. Note the MSC is independent of the number of cross-spectra from independent series that are used in the calculation. The value of the MSC does not change even though the coherent noise spectrum $G_{s_{1} s_{2}}(f)$ has been converted into the noise spectrum of two independent signals $N_{s_{1} s_{2}(t-D)}(f)$ and added to the spectrum of any uncorrelated random noise present. Its properties depend only on the number of segments/blocks, $n_{s}$.

The aligned and unaligned coherence procedure might have some application in the field of biomedical engineering. For example, in cardiovascular variability analysis, the degree of coupling between two time series can be estimated from the coherence function. This is called "setting a threshold level" by Faes ${ }^{58}$ and refers to deciding if the coherence measured is that of two random signals. Faes ${ }^{58}$ uses surrogate data analysis to assess the significance of the coherence function from two signals. A procedure based on using aligned and unaligned coherence functions might be more cost effective and efficient than the procedure tested by Faes. ${ }^{58}$

\section{Coherence with tones example}

In this section the application of the aligned and unaligned coherence procedure to a mixed spectrum consisting of discrete and continuous components will be discussed. The analysis of processes with mixed spectra is discussed by Priestly ${ }^{1}$ in chapter 8 . Many statistical approaches are reviewed. They all have trouble separating peaks in the continuous spectrum from peaks due to harmonic components. This is especially true if a narrow bandwidth is used. When the noise components become sharper and more peaked the noise component may resemble a harmonic, a complex periodic signal, or an almost periodic signal ( Bendat and Piersol ${ }^{13}$ Chapter 1). To demonstrate the application we first consider the measurement of a a correlated Gaussian stationary noise source with time history, $s(t)$, in a situation where the measurement is contaminated with a Gaussian stationary random noise, $n$, and a time series due to tones, $\chi(t)$ where the random noise, tonal noise, and the correlated noise are all independent. As an example, this could be the measurement of pressure at two points in the far field of a turbofan engine. The two measured time series $x_{1}(t)$ and $x_{2}(t)$ are

$$
\begin{aligned}
& x_{1}(t)=s_{1}(t)+n_{1}(t)+\chi_{1}(t) \\
& y_{2}(t)=s_{2}(t)+n_{2}(t)+\chi_{2}(t)
\end{aligned}
$$

The auto-and cross spectra for this case are 


$$
\begin{aligned}
G_{x_{1} y_{2}} & =G_{s_{1} s_{2}}(f)+N_{n_{1} n_{2}}(f)+\Xi_{\chi_{1} \chi_{2}}(f) \\
G_{x_{1} x_{1}} & =G_{s_{1} s_{1}}(f)+N_{n_{1} n_{1}}(f)+\Xi_{\chi_{1} \chi_{1}}(f) \\
G_{y_{2} y_{2}} & =G_{s_{2} s_{2}}(f)+N_{n_{2} n_{2}}(f)+\Xi_{\chi_{2} \chi_{2}}(f)
\end{aligned}
$$

where $N(f)$ is used to designate the spectrum of the noise, $G(f)$ is used to designate the spectrum of the coherent random signal and $\Xi(f)$ is used to designate the tonal auto and cross spectra.

The estimated MSC is then

$$
\left|\hat{\gamma}_{x y}(f)\right|^{2}=\frac{\left|G_{s_{1} s_{2}}(f)+N_{n_{1} n_{2}}(f)+\Xi_{\chi_{1} \chi_{2}}(f)\right|^{2}}{\left[G_{s_{1} s_{1}}(f)+N_{n_{1} n_{1}}(f)+\Xi_{\chi_{1} \chi_{1}}(f)\right]\left[G_{s_{2} s_{2}}(f)+N_{n_{2} n_{2}}(f)+\Xi_{\chi_{2} \chi_{2}}(f)\right]}
$$

Again, we start with the time series $x_{1}(t)$ and $y_{2}(t)$. However, we now delay the signal by an amount $D$ which is greater than the record length, $\mathrm{T}$. The signals analyzed are now

$$
\begin{aligned}
x_{1}(t) & =s_{1}(t)+n_{1}(t)+\chi_{1}(t) \\
y_{2}(t-D) & =s_{2}(t-D)+n_{2}(t-D)+\chi_{1}(t-D)
\end{aligned}
$$

The auto-and cross spectra for this case are

$$
\begin{aligned}
G_{x_{1} y_{2}} & =N_{n n}(f)+\Xi_{\chi_{1} \chi_{2}(t-D)}(f) \\
G_{x_{1} x_{1}} & =G_{s_{1} s_{1}}(f)+N_{n_{1} n_{1}}(f)+\Xi_{\chi_{1} \chi_{1}}(f) \\
G_{y_{2} y_{2}} & =G_{s_{2}(t-D) s_{2}(t-D)}(f)+N_{n_{2}(t-D) n_{2}(t-D)}(f)+\Xi_{\chi_{2}(t-D) \chi_{2}(t-D)}(f)
\end{aligned}
$$

The estimated MSC is then

$$
\left|\hat{\gamma}_{x y}(f, D \quad D>T)\right|^{2}=\frac{\left|N_{n n}(f)+\Xi_{\chi_{1} \chi_{2}(t-D)}(f)\right|^{2}}{\left[G_{s_{1} s_{1}}(f)+N_{n_{1} n_{1}}(f)+\Xi_{\chi_{1} \chi_{1}}(f)\right]\left[G_{s_{2}(t-D) s_{2}(t-D)}(f)+N_{n_{2}(t-D) n_{2}(t-D)}(f)+\Xi_{\chi_{2}(t-D) \chi_{2}(t-D)}(f)\right]}
$$

Since the noise auto and cross spectra are invariant with time delay and the tonal auto and cross spectra are invariant with time delay. We have

$$
\begin{aligned}
N_{s_{2} s_{2}} & =N_{s_{2}(t-D) s_{2}(t-D)} \\
G_{s_{1} s_{2}} & =G_{s_{1} s_{2}(t-D)} \\
G_{s_{2} s_{2}} & =G_{s_{2}(t-D) s_{2}(t-D)}
\end{aligned}
$$

Consequently, the estimated MSC is simplified to

$$
\left|\hat{\gamma}_{x y}(f, D \quad D>T)\right|^{2}=\frac{\left|N_{n n}(f)+\Xi_{\chi_{1} \chi_{2}}(f)\right|^{2}}{\left[G_{s_{1} s_{1}}(f)+N_{n_{1} n_{1}}(f)+\Xi_{\chi_{1} \chi_{1}}(f)\right]\left[G_{s_{2} s_{2}}(f)+N_{n_{2} n_{2}}(f)+\Xi_{\chi_{2} \chi_{2}}(f)\right]}
$$

Comparing the unaligned MSC (Eq. 46) and the aligned MSC (Eq. 50) we see that they have the same denominator. The numerator of the unaligned coherence differs in that the coherent noise spectrum has been changed into an incoherent noise spectrum. Consequently, in regions without tones the coherence will be that of two random uncorrelated signals measured using $n_{s}$ segments/blocks. Thus, the discussion in section VII for the MSC $\left|\hat{\gamma}_{x_{n} x_{b}}\right|^{2}$ apply to the unaligned MSC measured with tones if tones are not actually apparent in a particular frequency band.

Consequently, the regions without tones establish a level. All tones above this level will be observed. This procedure identifies all the tones in the measured spectrum above the background noise level set by the number of segments/blocks, $n_{s}$. The method using the aligned and unaligned coherence function with the averaged periodogram seems to be better than the tests discussed by Priestly. ${ }^{1}$ Note, that it has the same limitations in terms of resolution as any averaged periodogram procedure. Measurements are made over specific frequency bands. Tones are still lumped into these bands. However, tones lost in the broad band noise 
do appear. The procedure provides a new version of the turbofan engine noise spectrum. In addition, this is achieved without estimating parameters as is done by Sengupta and Kay, ${ }^{2}$ Kay and Nagesha ${ }^{3,4}$ and Ciuciu, Idier, and Giovannelli. ${ }^{5}$ It should also be noted that several methods are availbale to get line spectra that have higher resolution than the average periodogram procedure achieves (Cabrera, ${ }^{61}$ Sacchi, ${ }^{62} \mathrm{Ciuciu}^{5}$ ) and it is possible these could be used with the unaligned time histories to get the toneless cross-spectrum. Since the broadband noise has been essentially removed, it should not be necessay to use an average periodogram procedure.

The problems of time delay bias error and of confidence limits of coherence are discussed in texts and articles on random data analysis Bendat ${ }^{13-15}$ by Tukey, ${ }^{47}$ and in other texts on digital time series analysis Otnes and Enochson, ${ }^{63}$ Koopmans ${ }^{64}$ Priestley, ${ }^{65}$ and Brillinger.${ }^{66}$ These topics are crucial to the aligned and unaligned coherence method. In addition, the coherence of tones is well known. However, the use of the aligned and unaligned coherence for signal diagnosis is not discussed in these texts. The method is new and was only be developed for the present case where

- The signals analyzed are from turbofan engine noise and are a combination of random data and tones.

- The coherence is low but above the coherence of random noise.

We have shown how the cumulative distribution derived using he probability density function of the coherence derived by Goodman ${ }^{41}$ can be used to determine a P-percent confidence interval ( Eq. 11 ). Consequently, the coherence confidence interval of the unaligned coherence should correspond to this value

$$
\left.\hat{\gamma}_{x y}^{2}(f, D>T)\right|_{\text {unaligned }}=E_{I}=1-(1-P)^{1 /\left(n_{s}-1\right)}
$$

However, the estimated unaligned coherence provides more information since it is not based on any assumptions. For example, it accounts for any overlap used in the time series calculations and does not depend on the process being Gaussian or stationary. In addition, it is based on the same computational software and electronic hardware used to obtain the aligned coherence. In general this type of empirical approach is less approximate and has the advantage of being of broader applicability.

As a consequence using unaligned coherence functions i.e. coherence functions created by time shifting one time history more than the sample record length,$T_{d}$, we can see the coherence of the tones and measure the minimum coherence observable. Consequently, if provides a solution to two very difficult problems.

Thus using the unaligned coherence functions in addition to the aligned coherence functions provides a clear indication of how much of the result is due to random noise and how much of the result is due to correlated signals.

Jet noise measured between microphone separated by more than 10 degrees is at a radius of 150 feet uncorrelated between microphones. Consequently, the new technique when used with the three signal coherence method (Appendix B on page 33 ) to examine far field turbofan engine acoustic spectra produces using aligned signals acoustic spectra without jet noise but with coherent broadband noise and tones. With unaligned signals it produces spectra with only tones and completely random noise. This is an improvement since it separates tones from the turbofan engine noise and enables one to examine core and fan tonal noise sources. The new technique when used with the coherent output power method (Appendix C on page 35 ) to separate and identify correlated combustion noise in far field measurement turbofan engine noise provides more confidence in the results.

The procedure might be used to study broadband fan noise. One can imagine an active filter using the unaligned coherence to identify tones and to modify itself so that tones can be filtered from the signals. The method has utility on its own since in calculating the MSC of two random broad band noise source the two signals must be aligned to be detected. Consequently, using the aligned and unaligned coherence procedure can separate broad band noise from tonal noise providing the spectra do not overlap.

Under the old paradigm two time series $x(t)$ and $y(t)$ are regarded as "coherent" at a frequency $\mathrm{f}$ if $\hat{\gamma}_{x y}^{2}(f) \approx 1$ and incoherent if $\hat{\gamma}_{x y}^{2}(f) \approx 0$.

Under the new paradigm two time series $x(t)$ and $y(t)$ are regarded as "coherent" at a frequency $\mathrm{f}$ if $\left.|\hat{\gamma}|_{x y}^{2}\left(f, \tau_{1}=0\right)\right|_{\text {aligned }}$ is greater than $\left.|\hat{\gamma}|_{x y}^{2}\left(f, \tau_{1}>T_{d}\right)\right|_{\text {unaligned }}$ and incoherent if $\left.|\hat{\gamma}|_{x y}^{2}\left(f, \tau_{1}=0\right)\right|_{\text {aligned }}$ is less than or equal to $\left.|\hat{\gamma}|_{x y}^{2}\left(f, \tau_{1}>T_{d}\right)\right|_{\text {unaligned. }}$ This paradigm corresponds to the concept of "finding a threshold level in the coherence function for zero coherence" discussed by Faes. ${ }^{58}$ To the extent that translating the data beyond the block length, $T$, violates the traditional viewpoint on the proper procedure 
for coherence measurement, the use of aligned and unaligned coherence might be viewed as an elementary method of surrogate data analysis.

It is important to note that for the P\&W4098 test results reported herein sound wave travel time from the turbofan engine to the far field microphones introduces a time delay, $\tau_{o}=R / c_{o}$, where $R$ is the distance from the turbofan engine and $c_{o}$ is the sound propagation speed. As discussed, this time delay must be removed so that the Kulite signal and the far field microphone signal are aligned in order to see the correlated combustion noise. If the signals are analyzed as measured $\tau_{o}$ is greater than $T_{d}$ so the signals will be unaligned and the results will consist only of tones and a random noise coherence dependent on the number of averages, $n_{d}$.

\section{Results}

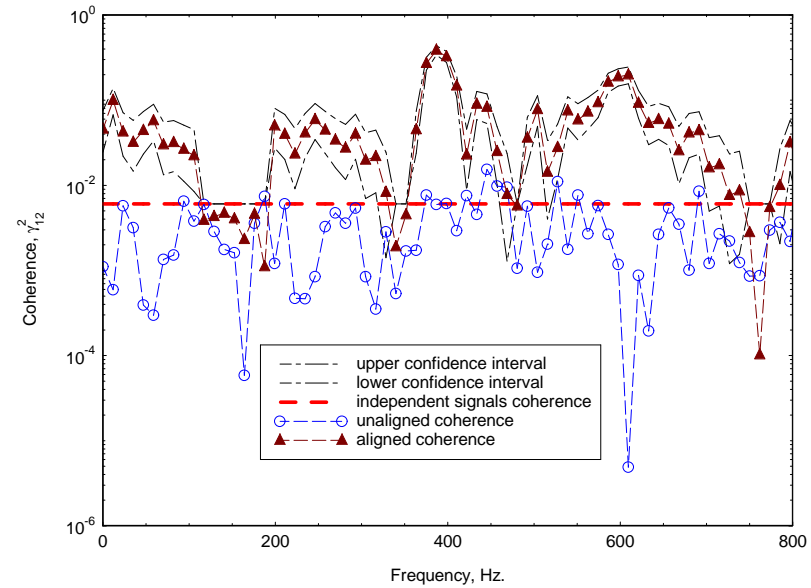

(a) $\mathrm{N} 1$ Corr $=1622$.

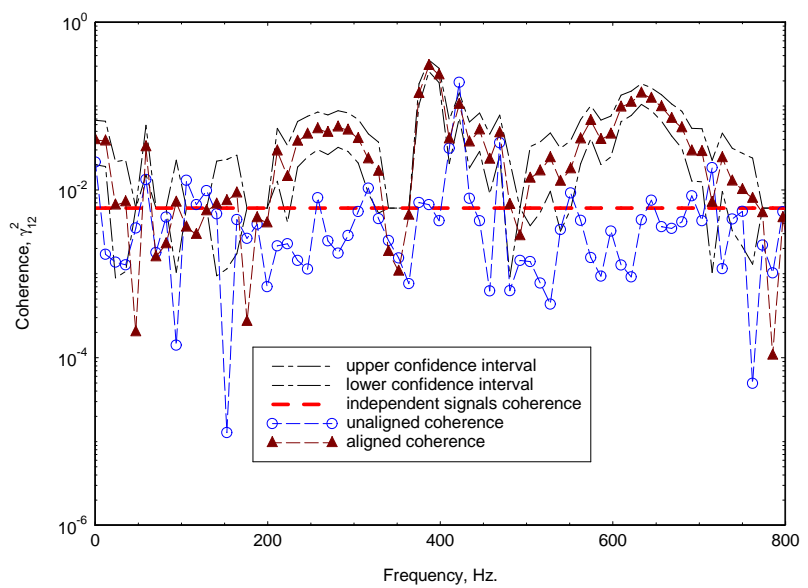

(b) N1 Corr $=1999$.

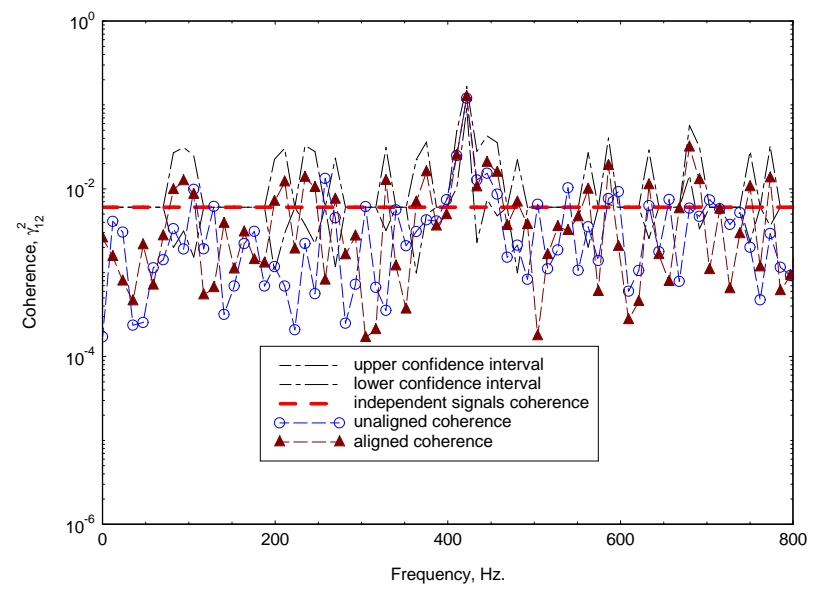

(c) N1 Corr $=2600$.

Figure 7. Aligned and unaligned coherence between Kulite (1) and (2).

\section{A. Kulite coherence}

Typical logarithmic plots of aligned and unaligned coherence between the two Kulites are shown in figure 7. Also shown is the confidence interval for the aligned coherence and the independent signal 95 percent coherence level, $\left|\hat{\gamma}_{x_{n} y_{n}}\right|^{2}$, calculated from Eq. 11. Note that as indicated in Fig. 4b, the confidence interval is 
narrow for coherence values near 1 and becomes much larger for lower coherence values.

As previously mentioned, the PW4098 combustor Kulite cross-spectra amplitude and phase plots resembled the cross-spectra used in the acoustic modal analysis of a YF102 combustor installed in a ducted test rig which was conducted by Karchmer Ref. ${ }^{7}$ This suggested that the observed structure in the coherence was due to a modal pattern. Consequently, using a model of the physics of pressure waves propagating in annular ducts as discussed by Tyler and Sofrin in a treatment of axial flow compressor noise ${ }^{8}$ a procedure was developed that uses the available Kulite data to obtain a descriptions of the combustion modes in the annular combustor. Results from this work were used to select the signals to be analyzed and to interpret the results in an attempt to identify combustion noise in the far field data. These broad peaks and dips in the coherence due to combustion duct modes are seen in figure 7 on the page before. Note, the aligned and unaligned coherence values (Eqs. 46 and 47) are well separated. The aligned coherence is well above the independent signal coherence line and the unaligned coherence plot. The unaligned coherence plot shows the presence of tones only at higher rpm values.

The coherent plane wave mode as determined by a model analysis is apparent in Fig. 7a between 0 and $200 \mathrm{~Hz}$ at $1662 \mathrm{rpm}$ (N1 CORR). However, it is not apparent in Fig. 7b between 0 and $200 \mathrm{~Hz}$ at 1999 rpm (N1 CORR) while coherent combustion noise is apparent at higher frequencies.

In Fig. 7c at $2600 \mathrm{rpm}(\mathrm{N} 1 \mathrm{CORR})$ the aligned and unaligned coherence are not well separated indicating one Kulite has failed (plot 7(c)). This failure is also seen in the auto-spectra plots shown in Miles ${ }^{6}$ where it is obeserved that Kulite 2 has failed.Note also that for this case the aligned and unaligned coherence are mostly below the independent signal coherence line. In calculating the independent signal coherence, $|\hat{\gamma}|_{x_{n} y_{n}}^{2}$, shown in the coherence plots a value of $n_{d}=500$ was used.

Using the aligned and unaligned coherence method one can observe sensor degradation, total sensor failure, or changes in the signal.

\section{B. Three signal coherence technique}

1. 1 far field microphones and 2 Kulites

The three signal coherence technique is described in Appendix B on page 33. It is applied to noise from a P\&W4098 turbofan engine using aligned and unaligned coherence functions (Eqs 49 and 51) in the threes signal cohernce technique. Typical results of applying the three signal and unaligned coherence technique are shown in figures 8 on the next page and figure 9 on page 26 . The example shown is for $1622 \mathrm{rpm}$ (N1 CORR). Results calculated using signals from 1 far field microphones and 2 Kulites are shown in figure 8 on the next page. Results calculated using signals from 3 far field microphones. are shown in Figure 9 on page 26 .

Figure 8 on the next page was created to see if the procedure would detect combustion noise. The number of translation points, D, used for each cross-spectrum so that the signals of interest would be in proper alignment is shown in the legend. To calculate the aligned $G_{33}$ spectrum a delay of 6839 time steps was used in calculating the cross-spectrum $G_{13}$, a delay of $G_{6134}$ time steps was used in calculating the $G_{23}$ cross-spectrum, and the $G_{12}$ cross-spectrum was used as measured. To calculate the unaligned $G_{33}$ spectrum a delay of 6323 time steps was used in calculating the cross spectrum $G_{12}$, and the $G_{13}$ and $G_{23}$ cross-spectrum were used as measured. The $\mathrm{D}=6839$ and $\mathrm{D}=6134$ time steps are in the range of the travel time from the turbofan to the microphones used. However, some attempt was made to find time intervals that maximized the coherence in the 0 to $200 \mathrm{~Hz}$. frequency band and those are used here.

Broad band combustion noise in the aligned power spectrum between 0 and $200 \mathrm{~Hz}$ is shown in Figure 8 on the following page. Tone noise appears in both the aligned and unaligned poser spectrum. The tone at one BPF ( first blade passing frequency) is especially noticeable. However, it is best shown in 


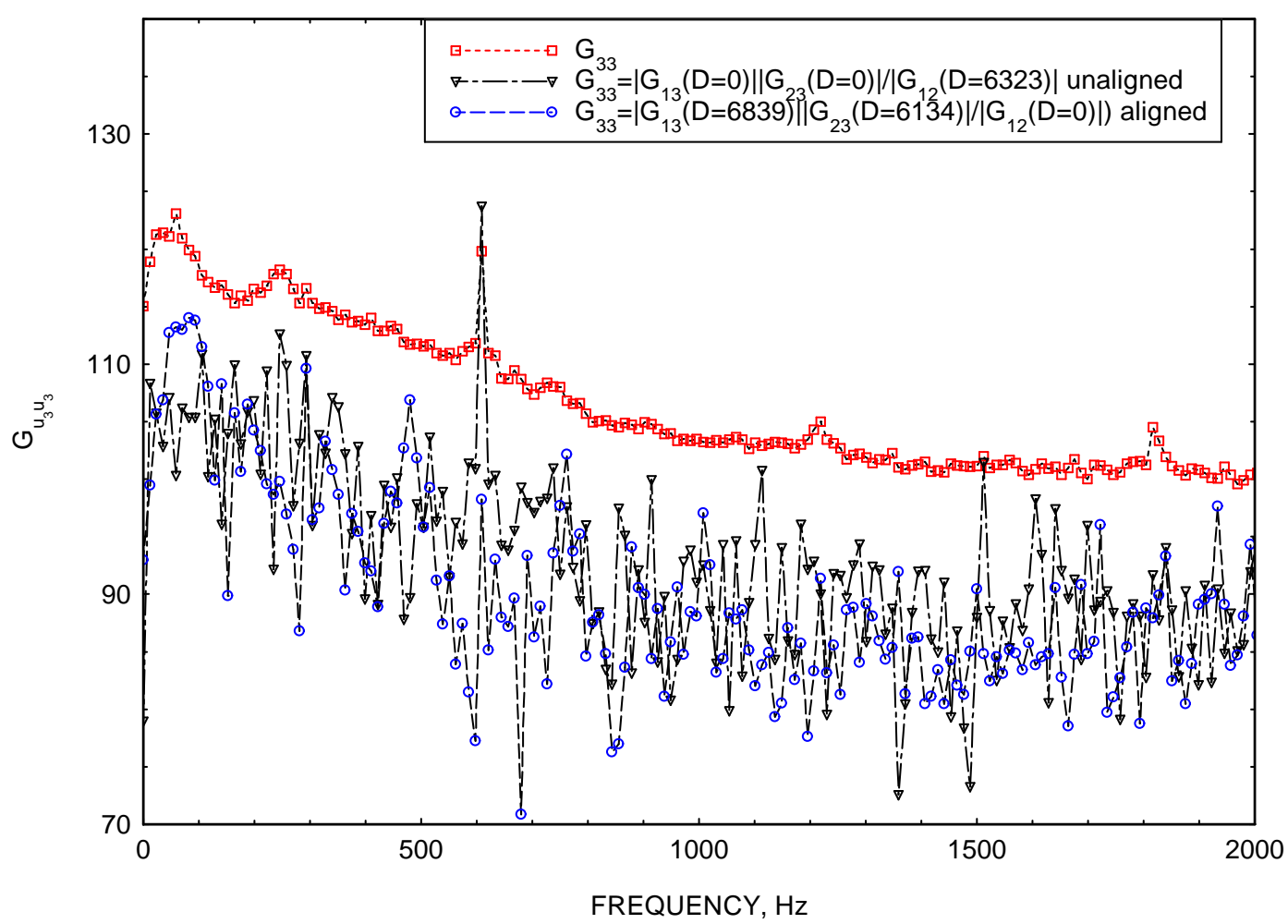

Figure 8. Three signal coherence technique using 1 far field microphone and two Kulites

the unaligned power spectrum where the broad band noise does not appear. Note that one can view tones approximately $10 \mathrm{~dB}$ below the measured auto spectrum in the 3 signal coherent aligned and unaligned power spectrum. In the unaligned 3 signal spectrum many tones previously unseen are now exposed to sight since the coherent broadband noise has been removed.

\section{3 far field microphones}

Figure 9 on the next page shows the three signal aligned and unaligned power spectra calculated using 3 far field microphones Three signal aligned and unaligned power spectra calculated using 3 far field microphones are shown in Figure 9 on the following page. The aligned coherence values were used as measured. The unaligned coherence values were found by dealying one signal 6323 time units with respect to the other. The jet noise is missing in the three signal aligned and unaligned power spectra to the extent that the microphones are far enough apart that the jet noise signals they receive are independent in this frequency range. This feature of jet noise is exploited by the three signal coherence technique using three far field microphones. The aligned and unaligned spectra from 0 to $500 \mathrm{~Hz}$. are well separated showing the presence of a coherent noise source. The plot clearly shows the tonal component due to the core and fan in the far field noise spectrum using the unaligned coherence (green dots). Above this spectrum is one made using the aligned coherence (blue dots). This clearly shows the broadband noise spectrum and the tonal component where it dominates. Above this is a broad band spectrum interrupted in places by tonal noise. The 1BPF, 


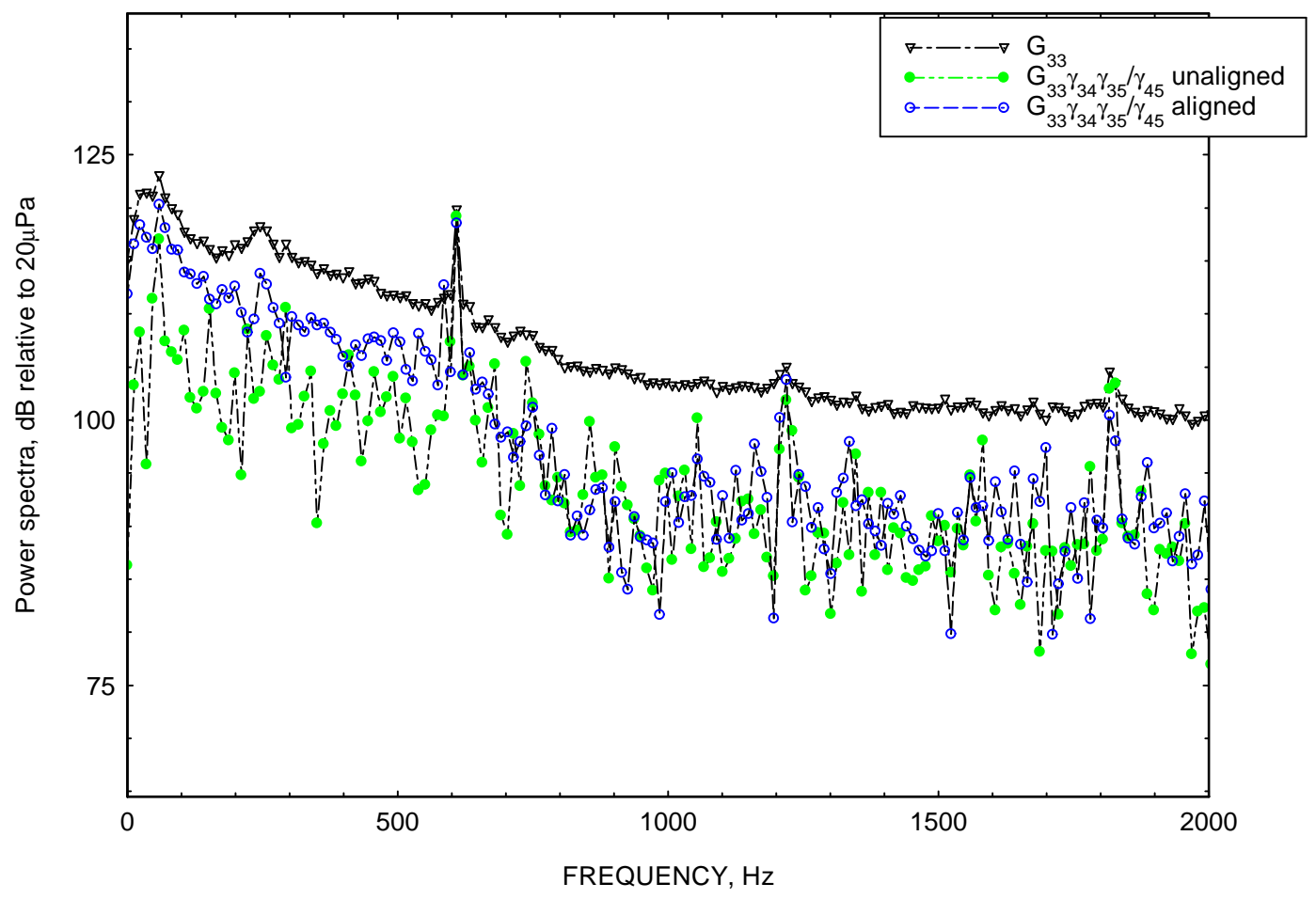

Figure 9. Three signal coherence technique using 1 far field microphone and two Kulites

2BPF, and $3 \mathrm{BPF}$ tones are clearly observable along with many other tones in the three signal aligned and unaligned power spectra. Note again, that one can view tones approximately $10 \mathrm{~dB}$ below the measured auto spectrum in the 3 signal coherent aligned and unaligned power spectrum. Similar behavior appears at other turbofan engine speeds.

\section{Coherent Output Power}

Coherent output power is described in Appendix C on page 35. Results for measurements using Kulite one and two with the 120 degree far field microphone (signal source 5) are presented in figure 10 on the following page for a test condition of $1622 \mathrm{rpm}$ (N1 CORR). To obtain the aligned coherent output power using Kulite one and signal source 5 , a time delay of $0.116875 \mathrm{sec}$ was used. To obtain the aligned coherent output power for Kulite two and signal source 5 a time delay of $0.128083 \mathrm{sec}$. was used. The plane wave combustion noise is apparent between 0 an $200 \mathrm{~Hz}$ in both aligned coherent output power spectra $\hat{\gamma}_{15}^{2} G_{55}$ and $\hat{\gamma}_{25}^{2} G_{55}$. Both spectra are clearly above their corresponding unaligned coherent output power spectra. The unaligned coherent output power spectra were obtained using the as measured signals with no time delay. Furthermore, the results suggest that combustion noise due to higher combustion duct modes is present near $300 \mathrm{~Hz}, 400$ $\mathrm{Hz}$, and $600 \mathrm{~Hz}$. Note that the noise floor is about 20dB below the measured auto-spectrum.

The corresponding aligned and unaligned coherence is shown in figure 11 on page 28. Also shown is the confidence interval for the aligned coherence and the independent signals 95 percent coherence level.

In calculating the coherence of random noise, $\hat{\gamma}_{x_{n} y_{n}}^{2}$, shown in the coherence plot a value of $n_{d}=500$ was used. Again the plane wave combustion noise is apparent between 0 an $200 \mathrm{~Hz}$ in both aligned coherence functions. Furthermore, the results suggest that combustion noise due to higher combustion duct modes is present near $300 \mathrm{~Hz}, 400 \mathrm{~Hz}$, and $600 \mathrm{~Hz}$.

\section{Discussion}

Table D on page 29 shows that using longer record lengths, $T_{\text {Total }}$, of 2 minutes or 5 minutes provide a significantly lower noise floor. Note that the coherent power noise floor is always a factor of 2 less than the coherence noise floor based on the three signal method. 


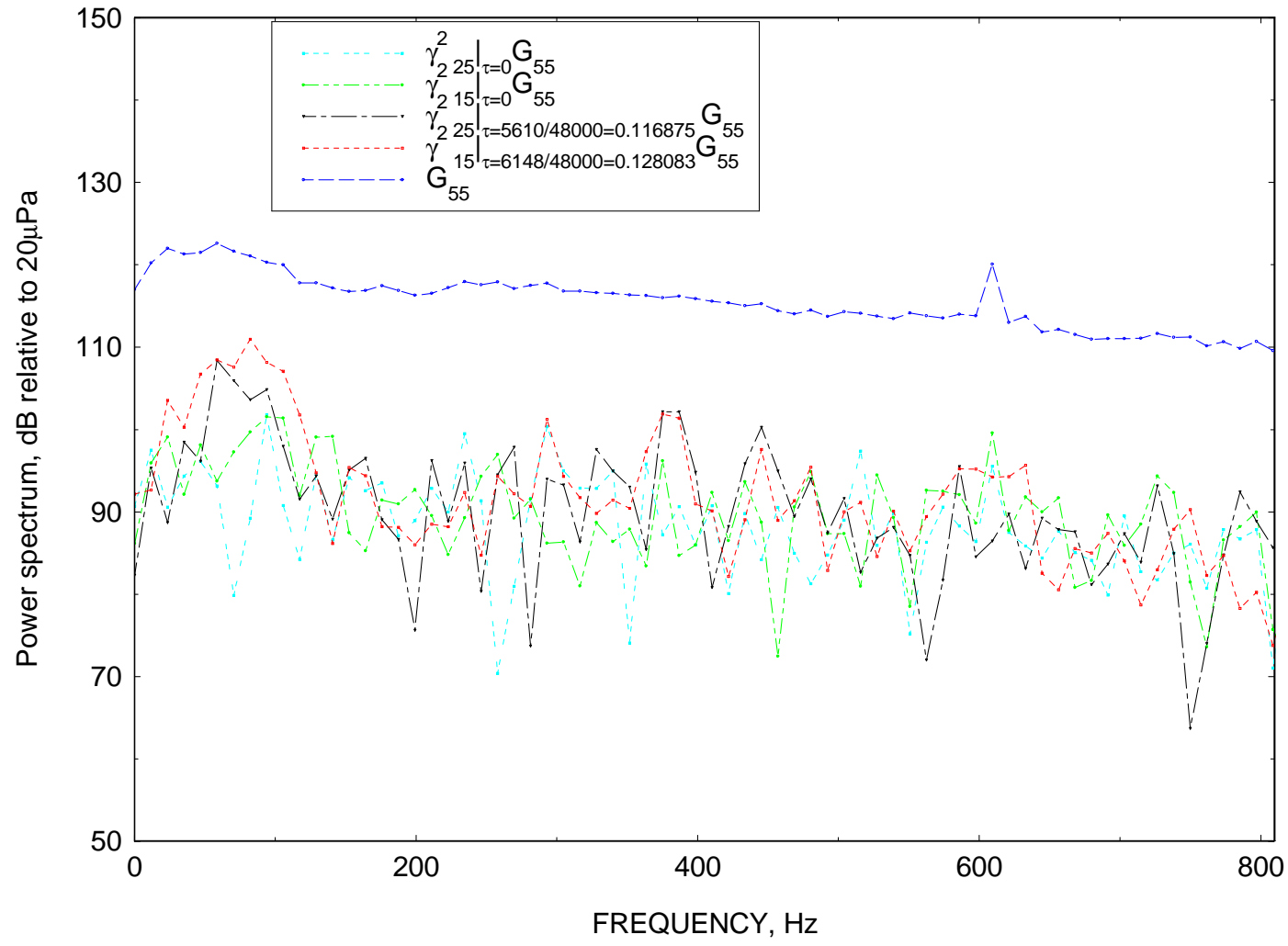

Figure 10. Total sound power, aligned and un-aligned coherent output power calculation for a test condition of $1622 \mathrm{rpm}$ (N1 CORR), using signals 1 (Kulite 1 at 127 Degrees clockwise from top dead center viewed from rear) and 5 (150 foot microphone at 120 Degrees). 


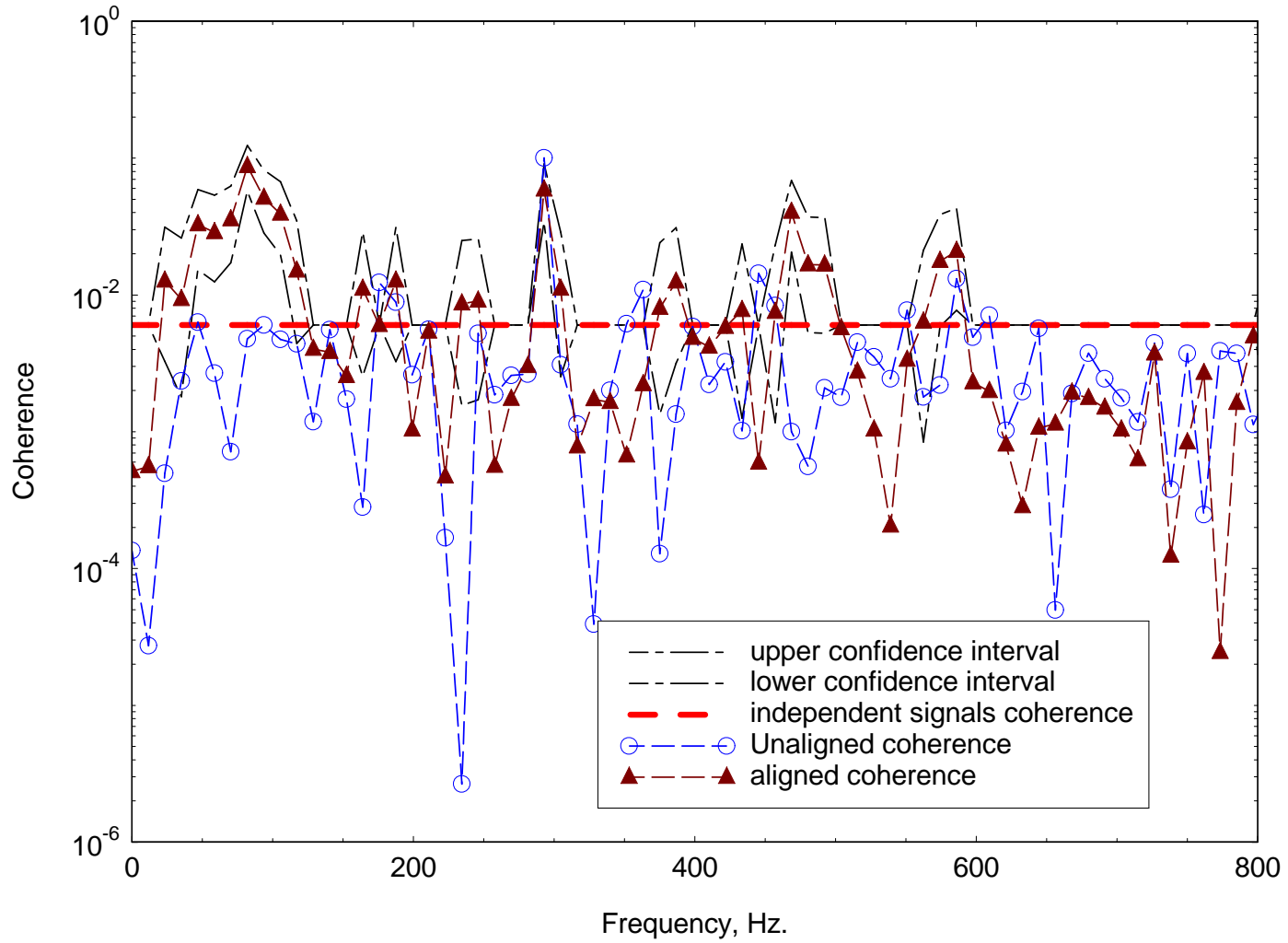

Figure 11. Aligned and un-aligned coherence calculation for a test condition at 1622 rpm (N1 CORR) using signals 1 (Kulite 1 at 127 Degrees clockwise from top dead center viewed from rear) and 5 (150 foot microphone at 120 Degrees). 
The standard procedure for coherence functions should be modified if the coherence is small. For a given number of independent segments/blocks, $n_{s}$ one can

- Compare it with the statistical theory value for the coherence of random noise.

- Compare it with coherence based on a simulation.

- Calculate the unaligned coherence function to see if the aligned coherence is greater than the coherence of two independent time series.

Table 2. Time series estimation parameters

$(r=48000$ samples/sec.,$N P=$ number of points in $\mathrm{FFT}=4096, \mathrm{P}=0.95$,

$B_{e}=r / N P=11.71875 H z ., n_{d}=B_{e} T_{T o t a l}=$ number of disjoint (indpendent) segments)

\begin{tabular}{|r|r|r|l|l|l|}
\hline $\begin{array}{r}\text { Total record length } \\
T_{\text {Total }}, \text { sec. }\end{array}$ & Overlap & $n_{d}$ & $\begin{array}{l}\gamma_{x_{n} y_{n}}= \\
1-(1-P)^{1 /\left(n_{d}-1\right)}\end{array}$ & $\begin{array}{l}\text { 3-signal } \\
\text { noise floor, dB }\end{array}$ & $\begin{array}{l}\text { Coherent Output Power } \\
\text { noise floor, dB }\end{array}$ \\
\hline 20 & 0 & 234 & 0.0127749 & -9.468 & -18.936 \\
\hline 20 & $50 \%$ & $\approx 468$ & 0.00639431 & -10.971 & -21.9421 \\
\hline 120 & 0 & 1406 & 0.00221992 & -13.3582 & -26.7164 \\
\hline 120 & $50 \%$ & $\approx 2812$ & 0.00106515 & -14.8629 & -28.7259 \\
\hline 300 & 0 & 3515 & 0.00085215 & -15.3474 & -30.6948 \\
\hline 300 & $50 \%$ & $\approx 7030$ & 0.000426105 & -16.8524 & -33.7048 \\
\hline
\end{tabular}

\section{E. Conclusions}

New technology has been developed based on a "paradigm" shift in the definition of coherence and applied to turbofan engine core noise diagnostics. The method provides a new procedure for estimating the degree of coupling between two time series. In addition, the procedure provides a new way to examine mixed spectra and identify tones mixed with broad band noise.

The aligned and unaligned coherence procedure used alone, used with the three signal coherence technique and used with the coherent power method provides new information.

- The combustor model structure can be observed due to the use of the aligned and unaligned combustor coherence.

- One can observe sensor degradation, total sensor failure, or changes in the signal.

- The underlying tonal structure which is buried under broadband noise and jet noise is clearly visible when the unaligned 3 signal coherent power is examined.

- Combustion noise can be observed in the far field using the aligned and unaligned coherence with the coherent power method.

It is expected that this method will improve turbofan source diagnostic tests and enable the identification of noise sources that are often confused with one another. Furthermore, the method might have application in the field of biomedical engineering whenever the degree of coupling between two time series needs to be evaluated. 
Table 3. $E_{I}$ threshold

\begin{tabular}{|c|c|c|c|c|}
\hline$n_{d}$ & $\begin{array}{l}80 \\
\text { percent }\end{array}$ & $\begin{array}{l}90 \\
\text { percent }\end{array}$ & $\begin{array}{l}95 \\
\text { percent }\end{array}$ & $\begin{array}{l}99 \\
\text { percent }\end{array}$ \\
\hline 4.000000 & .415196 & .535841 & .631597 & ".784557 \\
\hline 8.000000 & .205403 & .280314 & .348164 & .482053 \\
\hline 10.000000 & .163749 & .225736 & .283129 & .400516 \\
\hline 12.000000 & .136112 & .188869 & .238404 & .342067 \\
\hline 14.000000 & .116446 & .162322 & .205817 & .298296 \\
\hline 16.000000 & .101740 & .142304 & .181036 & .264358 \\
\hline 25.000000 & .064861 & .091482 & .117346 & .174596 \\
\hline 32.000000 & .050593 & .071585 & .092114 & .138046 \\
\hline 45.000000 & .035917 & .050986 & .065819 & .099372 \\
\hline 64.000000 & .025223 & .035889 & .046438 & .070490 \\
\hline 85.000000 & .018978 & .027039 & .035035 & .053348 \\
\hline 105.000000 & .015356 & .021897 & .028394 & .043314 \\
\hline 128.000000 & .012593 & .017967 & .023312 & .035612 \\
\hline 145.000000 & .011114 & .015863 & .020589 & .031474 \\
\hline 165.000000 & .009766 & .013942 & .018101 & .027690 \\
\hline 185.000000 & .008709 & .012436 & .016149 & .024717 \\
\hline 205.000000 & .007858 & .011224 & .014578 & .022321 \\
\hline 225.000000 & .007159 & .010227 & .013285 & .020349 \\
\hline 230.000000 & .007003 & .010005 & .012997 & .019909 \\
\hline 250.000000 & .006443 & .009205 & .011959 & .018325 \\
\hline 300.000000 & .005368 & .007671 & .009969 & .015284 \\
\hline 350.000000 & .004601 & .006576 & .008547 & .013109 \\
\hline 400.000000 & .004026 & .005754 & .007480 & .011475 \\
\hline 450.000000 & .003578 & .005115 & .006650 & .010204 \\
\hline 500.000000 & .003220 & .004604 & .005985 & .009186 \\
\hline 550.000000 & .002927 & .004185 & .005442 & .008353 \\
\hline 600.000000 & .002683 & .003837 & .004989 & .007659 \\
\hline 650.000000 & .002477 & .003542 & .004605 & .007071 \\
\hline 750.000000 & .002146 & .003069 & .003992 & .006130 \\
\hline 850.000000 & .001894 & .002708 & .003522 & .005410 \\
\hline 1050.000000 & .001533 & .002193 & .002852 & .004380 \\
\hline 2205.000000 & .000730 & .001044 & .001358 & .002087 \\
\hline 2405.000000 & .000669 & .000957 & .001245 & .001914 \\
\hline 2605.000000 & .000618 & .000884 & .001150 & .001767 \\
\hline 2805.000000 & .000574 & .000821 & .001068 & .001641 \\
\hline 3005.000000 & .000536 & .000766 & .000997 & .001532 \\
\hline 3205.000000 & .000502 & .000718 & .000935 & .001436 \\
\hline 3405.000000 & .000473 & .000676 & .000880 & .001352 \\
\hline 3605.000000 & .000446 & .000639 & .000831 & .001277 \\
\hline 3800.000000 & .000424 & .000606 & .000788 & .001211 \\
\hline 4030.000000 & .000399 & .000571 & .000743 & .001142 \\
\hline 6030.000000 & .000267 & .000382 & .000497 & .000764 \\
\hline 8030.000000 & .000200 & .000287 & .000373 & .000573 \\
\hline 10030.000000 & .000160 & .000230 & .000299 & .000459 \\
\hline 12030.000000 & .000134 & .000191 & .000249 & .000383 \\
\hline 14030.000000 & .000115 & .000164 & .000214 & .000328 \\
\hline 16030.000000 & .000100 & .000144 & .000187 & .000287 \\
\hline 18030.000000 & .000089 & .000128 & .000166 & .000255 \\
\hline 20030.000000 & .000080 & .000115 & .000150 & .000230 \\
\hline 22030.000000 & .000073 & .000105 & .000136 & .000209 \\
\hline
\end{tabular}

\section{References}

${ }^{1}$ M.B. Priestley. Spectral Analysis and Time Series. Elsevier Academic Press, 1981.

${ }^{2}$ Debasis Sengupta and Steven M. Kay. Parameter estimation and glrt detection in colored non-gaussian autoregressive processes. IEEE Transactions on Acoustics, Speech, and Signal Processing, 38 No. 10:1661-1676, October 1990.

${ }^{3}$ Stephen M. Kay and Venkatesh Nagesha. Estimation for processes with mixed spectra. In Proc. ICASSP93, pages 232-235, 1993.

${ }^{4}$ Steven M. Kay and Venkatesh Nagesha. Maximum likelihood estimations of signals in autoregressive noise. IEEE Transactions on signal processing, 42 No. 1:88-100, January 1994.

${ }^{5}$ Philippe Ciuciu, Jerome Idier, and Jean-Francois Giovannelli. Regularized estimation of mixed spectra using a circular gibbs-markov model. IEEE Transactions on Signal Processing, 49 No. 10:2202-2213, October 2001. 
${ }^{6}$ Jeffrey Hilton Miles. Validating coherence measurements using aligned and unaligned coherence functions. Technical Report AIAA-2006-1389, AIAA, 2006. presented at the 44th AIAA Aerospace Science Meeting, 9-12 Jan 2006 Reno Hilton Reno, Nevada.

${ }^{7}$ A. M. Karchmer. Acoustic modal analysis of a full scale annular combustor. Technical Report AIAA Paper 83-0760, NASA, 1983. NASA TM-83334.

${ }^{8}$ J.M. Tyler and T.G. Sofrin. Axial flow compressor noise studies. Transactions SAE, 70 :309-332, 1962.

${ }^{9}$ Saeed V. Vaseghi. Advanced Digital Signal Processing and Noise Reduction. John Wiley, 1996.

${ }^{10}$ G.R. Grimmett and D. R. Stirzaker. Probability and Random Processed. Oxford University Press, 1992.

${ }^{11}$ Emanuel Parzen. Stochastic Processes. Holden-Day, 1962.

${ }^{12}$ Samuel D. Stearns and DonR. Hush. Digital siganl analysis. Prentice-Hall, Inc., 1990.

${ }^{13}$ Julius S. Bendat and Allan G. Piersol. Measurement and Analysis of Random Data. John Wiley \&Sons, 1966.

${ }^{14}$ Julius S. Bendat and Allan G. Piersol. Random Data: Analysis and Measurement Procedures. John Wiley \&Sons,

1971. \&Sons, 1980

${ }^{15}$ Julius S. Bendat and Allan G. Piersol. Engineering Applications of Correlation and Spectral Analysis. John Wiley

${ }^{16} \mathrm{G}$. Clifford Carter. Coherence and time dealy estimation. Proceedings of the IEEE, 75 No. 2:236-255, February

1987.

${ }^{17}$ DM Halliday, JR Rosenberg, AM Amjad, P Breeze, BA Conway, and SF Farmer. A framework for the analysis of mixed time series/point process data-theory and application to the study of physiological tremor. Prog. Biophys Mol Biol, 64 No.:237-278, 1995.

${ }^{18}$ Gwilym M. Jenkins and Donald G. Watts. Spectral Analysis and it applications. Holden-Day, 1969.

${ }^{19}$ Samuel D. Stearns and Ruth A. David. Signal Processing Algorithms Using Fortran and C. Prentice-Hall, Inc.,

1993.

${ }^{20}$ Peter D. Welch. The use of fast fourier transform for the estimation of power spectra: A method based on time averaging over short, modified periodgrams. IEEE Transactions on audio and electroacoustics, AU-15 No.2:70-73, June 1967.

${ }^{21}$ James H. NMcClellan. Multidimensional spectral estimation. Proceedings of the IEEE, 70 No. 9:1029-1039, September 1982.

${ }^{22}$ G. Clifford Carter, Charles H. Knapp, and Albert H. Nuttall. Estimation of the magnitude-squared coherence function via overlapped fast fourier transform processing. IEEE Transactions on Audio and Electroacoustics, AU-21 No. 4:337-344, August 1973.

${ }^{23}$ G. Clifford Carter and Albert H. Nuttall. A brief summary of a generalized framework for power spectral estimation. Signal Processing, $2: 387-390,1980$.

${ }^{24}$ G. Clifford Carter and Albert H. Nuttall. On the weighted overlapped segment averaging method for power spectral estimation. Proceedings of the IEEE, 68 No. 10:1352-1354, October 1980.

${ }^{25}$ Albert H. Nuttall and G. Clifford Carter. A generalized framework for power spectral estimation. IEEE Transactions on Acoustics, Speech, and Signal Processing, ASSP-28No.3:334-335, June 1980.

${ }^{27}$ Christopher Bingham, Michael D. Godfrey, and John W. Tukey. Modern techniques of power spectrum estimation.

${ }^{26}$ Donald B. Percival and Andrew T. Walden. Spectral Analysis for Physical Applications. Cambridge University IEEE Transactions on audio and electroacoustics, AU-15 No. 2:56-66, June 1967.

${ }^{28}$ M. J. Hinich and C. S. Clay. The application of the discrete fourier transform in estimation of power spectra coherence and bispectra of geophysical data. Rev. Geophys., 6 :347-363, August 1968. benignus1969 ref. 5.

${ }^{29}$ Albert H. Nuttall and Clifford Carter. Spectral estimation using combined time and lag weighting. Proceedings of the IEEE, 70 No. 9:1115-1125, September 1982.

${ }^{30}$ David R. Brillinger. Time Series Data Analysis and Theory -Expanded Edition. Holden-Day, 1981. ISBN:0-8162-

1150-7.

${ }^{31}$ Steven M. Kay and Jr. Stanley Lawrence Marple. Spectrum analysis - a modern perspective. Proceedings of the IEEE, 69 No. 11:1380-1420, November 1981.

${ }^{32} \mathrm{E}$ H Scannell Jr. and G C Carter. Confidence bounds for magnitude-squared coherence estimates. IEEE Trans Acoust Speech, and Signal Processing, ASSP-26 No. 5:475-477, October 1978.

${ }^{33} \mathrm{E}$ H Scannell Jr. and G C Carter. Confidence bounds for magnitude-squared coherence estimates. In Proc. IEEE Int. Conf. Acoust., Speech, Signal Processing, pages 670-673, 1978.

${ }^{34} \mathrm{G}$. Clifford Carter. Receiver operating characteristics for a linearly thresholded coherence estimation detector. IEEE Transactions on Acoustics, Speech, and Signal Processing, ASSP-25 :90-92, February 1977.

${ }^{35} \mathrm{G}$. Clifford Carter and Charles H. Knapp. Coherence and its estimation via the partitioned modified chirp-z transform. IEEE Transactions on Acoustics, Speech, and Signal Processing, ASSP-23 No. 3:257-264, June 1977.

${ }^{36}$ Allan Piersol. Time delay estimation using phase data. IEEE Transactions on Acoustics, Speech, and Signal Processing, ASSP-29 No. 3:471-477, June 1981.

${ }^{37}$ K.A. Brownlee. Statistical Theory and Methodology In Science and Engineering. John Wiley, 1960.

${ }^{38}$ T.W. Anderson. An introduction to Multivariate Statistical Alnalysis. John Wiley, 1958.

${ }^{39}$ Franklin A. Graybill. An Introduction to Linear Statistical Models. McGraw-Hill, 1961.

${ }^{40}$ Harald Cramer. Mathematical Methods of Statistics. Princeton University Press, 1966.

${ }^{41}$ N. R. Goodman. On the joint estimation of the spectra, cospectrum, and quadrature spectrum of a two-dimensional statioary gaussian process. Technical Report Scientific paper 10, Engineering Statistics Laboratory of New York University, 1957. AD-134919.

${ }^{42}$ E. J. Hannan. Multiple Time Series. John Wiley, 1970. 
${ }^{43}$ Fritz Oberhettinger. Hypergeometric functions. In Milton Abramowitz and Irene A. Stegun, editors, Handbook of Mathematical Functions, chapter 9, pages 555-566. Dover Publications, 1964.

${ }^{44}$ Shou-Yan Wang, Xuguang Liu, John Yianni, R. Christopher Miall, Tipu Z. Aziz, and John F. Stein. Optimising coherence estimation to assess the functional correlation of tremor-related activity between the subthalamic nucleus and the forearm muscles. Journal of Neurosciece Methods, 136 No.:197-205, 2004.

${ }^{45}$ Shou Yan Wang and MengXing Tang. Exact confidence inverval for magnitude-squared coherence estimates. IEEE Signal Processing Letters, 11 No. 3:326-329, March 2004.

${ }^{46}$ Albert H. Nuttall. Invariance of distribution of coherence estimate to second-channel statistics. IEEE Transactions on Acoustics, Speech, and Signal Processing, ASSP-29 No. 1:120-122, February 1981.

${ }^{47} \mathrm{John}$ W. Tukey. An introduction to the calculations of numerical spectrum analysis. In Bernard Harris, editor, Spectral Amalysis of Time Series, pages 25-46. Wiley and Sons, New York, 1967.

${ }^{48}$ M. R. Foster and N. J. Guinzy. The coefficient of coherence: Its estimation and use in geophyiscal dagta processing. Geophysics, 32 No. 4:602-616, August 1967.

${ }^{49} \mathrm{~V}$. A. Benignus. Estimation of the coherence spectrum and its confidence interval using the fast fourier transform. IEEE Trans. Audio Electroacoustics, AU-17 No. 2:145-150, June 1969.

${ }^{50}$ Leo J/ Tick. Estimation of coherency. In Bernard Harris, editor, Spectral Amalysis of Time Series, pages 133-152. Wiley and Sons, New York, 1967.

${ }^{51}$ A. F. Seybert and J. F. Hamilton. Time delay bias errors in estimating frequency response and coherence funcions. Journal of Sound and Vibration, 60 No. 1:1-9, 1978.

${ }^{52}$ G. C. Carter. Bias in magnitude-squared coherence estimation due to misalignment. IEEE Trans. ASSP, 28 No. 1:97-99, February 1980.

${ }^{53}$ Wilbur B. Davenport and William Root. An introduction to the Theory of Random Signals and Noise. McGraw-Hill, 1958.

${ }^{54}$ William G. Halvorsen and Julius S Bendat. Noise source identification using coherent output power spectra. Sound and Vibration, 9No.:15-24, 1975.

${ }^{55}$ L.H. Koopmans. The Spectral Analysis of Time Series. Academic Press, 1974.

${ }^{56}$ L. J. Tick. Estimation of coherence. In Bernard Harris, editor, Spectral Analysis of Time Series, pages 133-152. John Wiley and Sons, New York, 1967. Proceedings of an Advanced Seminar Conducted by the Mathematics Research Center, United States Army and the Statistics Deparartment at the University of Wisconsin, Madison October 3-5, 1966.

${ }^{57} \mathrm{M}$. W. Trethewey and H. A. Evensen. Time-delay bias errors in estimating frequency response and coherence functions from windowed samples of continuous and transient signals. Journal of Sound and Vibration, 97 No. 4:531-540, 1984.

${ }^{58}$ Luca Faes, Gian Domenico Pinna, Alberto Porta, Roberto Maestri, and Giandomenico Nollo. Surrogate data analysis for assessing the significance of the coherence function. IEEE Transactions on Biomedical Engineering, 51 No. 7:11561166, July 2004.

${ }^{59}$ James Theiler, Stephen Eubank, Andre Longtin, Bryan Galriian, and J. Doyne Farmer. Testing for nonlinearity in time series: the method of surrogate data. Physica D, $\mathbf{5 8}$ No.:77-94, 1992.

${ }^{60}$ Thomas Schreiber and Andreas Schmitz. Surrogate time series. Physica D, 142 No.:346-382, 2000.

${ }^{61}$ Sergio D. Cabrera and Thomas W. Parks. Extrapolation and spectral estimation with iterative weighted norm modification. IEEE Transactions on Signal Processing, 39 No. 4:842-850mebub2, April 1991.

${ }^{62}$ Mauricio D. Sacchi, Tadeusz J. Ulrych, and Colin J. Walker. Interpolation and extrapolation using a high-resolution discrete fourier transform. IEEE Transactions on Signal Processing, 46 No. 1:31-38, Janurary 1998.

${ }^{63}$ Robert K. Otnes and Loren Enochson. Digital Time Series Analysis. John Wiley and Sons, New York, 1972. coherence confidence limits p351.

${ }^{64}$ L. H. Koopmans. The Spectral Analysis of Time Series. Academic Press, 1974.

${ }^{65}$ M. B. Priestley. Spectral Analysis and Time Series. Elsevier Academic Press, 1981.

${ }^{66}$ David R. Brillinger. Time Series Data Analysis and Theory. Holden-Day, 1981.

${ }^{67}$ Meyer Reshotko, Allen Karchmer, Paul F. Penko, and Jack G. McArdle. Core noise measurements on a yf-102 turbofan engine. J. Aircraft, 14 No. 7:611-612, July 1977. NASA TM X-73587.

${ }^{68}$ Meyer Reshotko, Allen Karchmer, and Paul F. Penko. Core noise measurements on a yf-102 turbofan engine. AIAA Paper 77-21, Jan 1977.

${ }^{69}$ Eugene A. Krejsa. New technique for the direct measurement of core noise from aircraft engines. Technical Report TM-82634, NASA, 1981.

${ }^{70}$ Edvard Grande. Exhaust noise field generated in the jt8d core engine- noise floor presented by the internal noise sources. J. Acoust. Soc. Am., 55 No.1:30-34, January 1974

${ }^{71}$ U. Michel H.A. Siller, F. Arnold. Invesigation of aero-engine core-noise using a phased microphone array. Technical Report AIAA 2001-2269, 7th AIAA/CEAS Aeroacoustics Conference, 2001.

${ }^{72}$ Vincent Tesson, Michael J. Doty, and Dennis K MacLaughlin. Experimental investigation of jet noise and core noise using a small gas turbine engine. Technical Report AIAA 2002-2558, 8th AIAA/CEAS Aeroacoustics Conference, 2002.

${ }^{73}$ J. Y. Chung, Malcolm J. Crocker, and James F. Hamiton. Measurement of frequency response and the multiple coherence function of the noise-generation system of a diesel engine. J. Acoust. Soc. Am., 58 No. 3:636-642, September 1975.

${ }^{74}$ J. Y. Chung. Rejection of flow noise using a coherence function method. J. Acoust. Soc. Am., 62 No. 2:388-395, August 1977 .

${ }^{75}$ Belur N. Shivashankara. High bypass ratio engine noise component separation by coherence technique. J. Aircraft, 20 No. 3:236-242, March 1983. AIAA 81-2054, Aeroacoustics Conference, 7th, Palo Alto, CA Oct. 5-7, 1981. 
${ }^{76}$ J.S. Hsu and K. K. Ahuja. A coherence-based technique to separate ejector internal mixing noise from farfield measurements. Technical Report AIAA-98-2296, A98-30856,AIAA/CEAS Aeroacoustics Conference, 4th (19th AIAA Aeroacoustics Conference), Toulouse, France, June 2-4, 1998, Collection of Technical Papers. Pt. 2; Reston, VA AIAA 1998 p. 537-551, Georgia Tech Research Institute, Georgia Institute of Technology GTRI/AERO Atlanta, Georgia 30032-0844, 1998.

${ }^{77}$ R. W. Stoker, K. K. Ahuja, and J. HJsu. Separation of wind-tunnel background noise and wind noise from automobile interior measurements. Technical Report AIAA-96-1763,A96-30873, Georgia Tech Research Institute, Georgia Institute of Technology GTRI/AERO Atlanta, Georgia 30032-0844, 1996. AIAA and CEAS, Aeroacoustics Conference, 2nd, State College, PA May 6-8, 1996.

${ }^{78}$ A. Michalke, F. Arnold, and F. Holste. On the coherence of the sound field in a circular duct with uniform mean flow. Journal of Sound and Vibration, 190 No. 2:261-271, 1996.

${ }^{79} \mathrm{~K}$. K. Ahuja. Designing clean jet noise facilities and making accurate jet noise measurements. Technical Report AIAA 2003-0706, Georgia Institute of Technology GRRI/ATASL Atlanta, GA 30332, 2003. Invited paper at 41st AIAA Aerospace Sciences Meeting.

${ }^{80}$ Allen M. Karchmer. Identification and measurement of combustion noise from a turbofan engine using correlation and coherence techniques. Technical Report NASA TM-73747, NASA, 1977. Doctor of Philosphy thesis E-9319.

${ }^{81}$ A.M. Karchmer, M. Reshotko, and F.J. Montegani. Measurement of far field combustion noise from a turbofan engine using coherence functions. Technical Report AIAA Paper 77-1277, NASA, Oct. 1977. NASA TM-73748,N77-33163.

\section{Fundamentals}

\section{A. Previous Work}

Core noise was studied at the NASA Glenn Research Center when it was known as the NASA Lewis Research Center using a AVCO-Lycoming YF102 byReshotko, Karchmer, Penko, and McArdle ${ }^{67}, 68$ and Krejsa. ${ }^{69}$ Grande $^{70}$ studied JT8D core noise Ref. . He found a strong peak at approximately $150 \mathrm{~Hz}$ which he attributed to the rotational frequency of the high-pressure spool and the second harmonic of the rotational frequency of the low-pressure spool. Sillier, Arnold and Michael ${ }^{71}$ used a phased microphone array to investigate aero-engine core-noise of a BR700 aero-engine. Lesson, Dotty and McLaughlin ${ }^{72}$ studied jet noise and Core noise from a small gas turbine APU type engine.

\section{B. Three signal coherence technique}

The three signal coherence technique was developed by Chung ${ }^{73,74}$ for flow noise rejection. A similar technique was developed and used byKrejsa. ${ }^{69}$ The three signal coherence technique was used byShivashankara ${ }^{75}$ to study core noise in a Pratt and Whitney JT9D. It was used by Hsu and Ahuja ${ }^{76}$ to separate ejector internal mixing noise from far field measurements and by Stoker, Ahuja, and $\mathrm{Hsu}^{77}$ to separate wind-tunnel background noise and wind noise from automobile interior noise measurements. It was used byMichalke,Arnold and Holste ${ }^{78}$ to study sound in a circular duct with mean flow .

The complex coherence function is the normalized cross spectral density

$$
\gamma_{x y}(f)=\frac{G_{x y}(f)}{\sqrt{G_{x x}(f) G_{y y}(f)}}
$$

where $G_{x y}(f)$ is the cross-spectral density at frequency $f$ between acoustic signals $x(t)$ and $y(t)$ with auto spectra $G_{x x}$ and $G_{y y}$. Another coherence function is the magnitude-squared coherence, $\gamma_{x y}^{2}$. where

$$
\gamma_{x y}^{2}(f)=\frac{\left|G_{x y}(f)\right|^{2}}{G_{x x}(f) G_{y y}(f)}
$$

The technique is developed as follows. We assume each of the three external microphones pick up the core noise and uncorrelated external noise from the jet.

Then

$$
\begin{aligned}
& y_{1}(t)=u_{1}(t)+\ell(t) \\
& y_{2}(t)=u_{2}(t)+m(t) \\
& y_{3}(t)=u_{3}(t)+n(t)
\end{aligned}
$$


Since the time histories $u_{1}(t), u_{2}(t)$, and $u_{3}(t)$ are due to core noise the ordinary coherence function between pairs is assumed to be unity.

$$
\begin{aligned}
\gamma_{u_{1} u_{2}}^{2}(f) & =\frac{\left|G_{u_{1} u_{2}}(f)\right|^{2}}{G_{u_{1} u_{1}}(f) G_{u_{2} u_{2}}(f)}=1 \\
\gamma_{u_{1} u_{3}}^{2}(f) & =\frac{\left|G_{u_{1} u_{3}}(f)\right|^{2}}{G_{u_{1} u_{1}}(f) G_{u_{3} u_{3}}(f)}=1 \\
\gamma_{u_{2} u_{3}}^{2}(f) & =\frac{\left|G_{u_{2} u_{3}}(f)\right|^{2}}{G_{u_{2} u_{2}}(f) G_{u_{3} u_{3}}(f)}=1
\end{aligned}
$$

The core noise and the external noise are uncorrelated. Thus

$$
G_{u_{1} \ell}(f)=G_{u_{2} m}(f)=G_{u_{3} n}(f)=0
$$

Further more the external jet noise at each microphone for an angular separation greater than 10 degrees is uncorrelated due to the distributed nature of jet mixing noise. Experimental results showing this are given byThuja. ${ }^{79}$ Thus

$$
G_{\ell m}(f)=G_{\ell n}(f)=G_{m n}(f)=0
$$

The ordinary coherence function between microphones 1 and 2 is calculated as follows using Eq. (4)

$$
\begin{aligned}
\gamma_{y_{1} y_{2}}^{2}(f) & =\frac{\left|G_{u_{1} u_{2}}(f)\right|^{2}}{\left(G_{u_{1} u_{1}}(f)+G_{\ell \ell}(f)\right)\left(G_{u_{2} u_{2}}(f)+G_{m m}(f)\right)} \\
& =\frac{G_{u_{1} u_{1}}(f) G_{u_{2} u_{2}}(f)}{\left(G_{u_{1} u_{1}}(f) G_{u_{2} u_{2}}(f)+G_{\ell \ell}(f) G_{u_{2} u_{2}}(f)+G_{m m}(f) G_{u_{1} u_{1}}(f)+G_{\ell \ell}(f) G_{m m}(f)\right)} \\
& =\frac{1}{1+\frac{G_{\ell \ell}(f)}{G_{u_{1} u_{1}}(f)}+\frac{G_{m m}(f)}{G_{u_{2} u_{2}}(f)}+\frac{G_{\ell \ell}(f) G_{m m}(f)}{G_{u_{1} u_{1}}(f) G_{u_{2} u_{2}}(f)}}
\end{aligned}
$$

To simplify equation (7) we define

$$
\begin{aligned}
a & =\frac{G_{\ell \ell}(f)}{G_{u_{1} u_{1}}(f)}=\frac{G_{y_{1} y_{1}}(f)-G_{u_{1} u_{1}}(f)}{G_{u_{1} u_{1}}(f)} \\
b & =\frac{G_{m m}(f)}{G_{u_{2} u_{2}}(f)}=\frac{G_{y_{2} y_{2}}(f)-G_{u_{2} u_{2}}(f)}{G_{u_{2} u_{2}}(f)}
\end{aligned}
$$

Then the ordinary coherence function $\gamma_{y_{1} y_{2}}^{2}$ becomes

$$
\gamma_{y_{1} y_{2}}^{2}=\frac{1}{1+a+b+a b}
$$

Using the third microphone and letting

$$
c=\frac{G_{n n}(f)}{G_{u_{3} u_{3}}(f)}=\frac{G_{y_{3} y_{3}}(f)-G_{u_{3} u_{3}}(f)}{G_{u_{3} u_{3}}(f)}
$$

we obtain by pairing off the microphones

Microphone 1 and 2:

$$
\gamma_{y_{1} y_{2}}^{2}=\frac{1}{1+a+b+a b}
$$

Microphone 1 and 3:

$$
\gamma_{y_{1} y_{3}}^{2}=\frac{1}{1+a+c+a c}
$$


Microphone 2 and 3:

$$
\gamma_{y_{2} y_{3}}^{2}=\frac{1}{1+b+c+b c}
$$

Substituting Eqs (8), (9) and (11) for $a, b$, and $c$ and solving for $G_{u_{1} u_{1}}, G_{u_{2} u_{2}}$, and $G_{u_{3} u_{3}}$ yields

$$
\begin{aligned}
G_{u_{1} u_{1}}(f) & =G_{y_{1} y_{1}}(f) \frac{\gamma_{y_{1} y_{2}}(f) \gamma_{y_{1} y_{3}}(f)}{\gamma_{y_{2} y_{3}}(f)} \\
G_{u_{2} u_{2}}(f) & =G_{y_{2} y_{2}}(f) \frac{\gamma_{y_{1} y_{2}}(f) \gamma_{y_{2} y_{3}}(f)}{\gamma_{y_{1} y_{3}}(f)} \\
G_{u_{3} u_{3}}(f) & =G_{y_{3} y_{3}}(f) \frac{\gamma_{y_{1} y_{3}}(f) \gamma_{y_{2} y_{3}}(f)}{\gamma_{y_{1} y_{2}}(f)}
\end{aligned}
$$

By using the definition of the complex coherence function as given by Eq. (1). Then

$$
\begin{aligned}
& W_{1 u_{1} u_{1}}(f)=\frac{G_{y_{1} y_{2}}(f) G_{y_{1} y_{3}}(f)}{G_{y_{2} y_{3}}(f)} \\
& W_{1 u_{2} u_{2}}(f)=\frac{G_{y_{1} y_{2}}(f) G_{y_{2} y_{3}}(f)}{G_{y_{1} y_{3}}(f)} \\
& W_{1 u_{3} u_{3}}(f)=\frac{G_{y_{1} y_{3}}(f) G_{y_{2} y_{3}}(f)}{G_{y_{1} y_{2}}(f)}
\end{aligned}
$$

where $W_{1 u_{1} u_{1}}(f), W_{1 u_{2} u_{2}}(f)$, and $W_{1 u_{2} u_{2}}(f)$ are complex.

The form thatKrejsa ${ }^{69}$ used is obtained by assuming that the sound field is coherent.

Then

$$
\begin{aligned}
& G_{u_{1} u_{1}}(f)=\left|W_{1 u_{1} u_{1}}(f)\right|=\mid \frac{G_{y_{1} y_{2}}(f)|| G_{y_{1} y_{3}}(f) \mid}{\left|G_{y_{2} y_{3}}(f)\right|} \\
& G_{u_{2} u_{2}}(f)=\left|W_{1 u_{2} u_{2}}(f)\right|=\frac{\left|G_{y_{1} y_{2}}(f)\right|\left|G_{y_{2} y_{3}}(f)\right|}{\left|G_{y_{1} y_{3}}(f)\right|} \\
& G_{u_{3} u_{3}}(f)=\left|W_{1 u_{3} u_{3}}(f)\right|=\frac{\left|G_{y_{1} y_{3}}(f)\right|\left|G_{y_{2} y_{3}}(f)\right|}{\left|G_{y_{1} y_{2}}(f)\right|}
\end{aligned}
$$

\section{Coherent output power}

In the past, methods have been developed using spectrum analysis techniques to characterize acoustic signals from different sources mixed with noise. Bendat ${ }^{13-15}$ discusses these methods . Among the methods discussed is using coherent output power spectra for noise source identification. The application of this technique that is of interest is the use of coherent output power spectra to separate and identify correlated combustion noise in far field measurements of turbofan engine noise. Karchmer ${ }^{80}$ and Karchmer, Reshotko, and Montegani.,${ }^{81}$ use the coherence function calculated from internal microphone measurements of fluctuating pressures in the combustor and far field acoustic pressures to determine the correlated combustion noise of a YF102 turbofan engine at far field locations by calculating the coherent output power spectrum. A typical results showed the coherence measured between the combustor pressure and the 120 degree far-field acoustic pressure had a roughly Gaussian shaped distribution in the range $0 \mathrm{~Hz}$ to $200 \mathrm{~Hz}$ with a peak near $125 \mathrm{~Hz}$ and a maximum value at $30 \%$ speed of 0.38 and at $43 \%$ speed a maximum value of $0.5\left(\right.$ Karchmer $^{80}$ Figs 39 and 48). The corresponding combustion coherence output power spectrum for the $43 \%$ speed case have a peak near 125 Hertz and a generally dome like shape. The peak is about $20 \log (0.5)=-6.02 \mathrm{~dB}$ below the peak of the far-field spectrum at $120 \mathrm{~Hz}$. The dome edges are $20 \mathrm{~dB}$ down at $40 \mathrm{~Hz}$ and $200 \mathrm{~Hz}$. The basic formulation for the coherent output power spectrumis presented by Bendat and Piersol. ${ }^{15} \mathrm{We}$ assume that $s_{F}$ is the output of a constant parameter linear system with weighting function $h(\tau)$ and frequency response $H(f)$. The output of the system is given by the convolution integral 


$$
s_{F}(t)=\int_{0}^{\infty} h(\tau) s_{c}(t-\tau) d \tau .
$$

Then the far field combustion noise spectrum, $G_{s_{F} s_{F}}(f)$, and the combustion noise cross spectrum between the far field signal and the Kulite signal, $G_{s_{F} s_{c}}(f)$ are related to the Kulite signal as follows:

$$
\begin{aligned}
G_{s_{F} s_{F}}(f) & =|H(f)|^{2} G_{s_{c} s_{c}}(f) \\
G_{s_{F} s_{c}}(f) & =H(f) G_{s_{c} s_{c}}(f)
\end{aligned}
$$

Here the cross power spectrum between the combustor Kulite signal, $x(k)$, and the far field microphone signal, $y(k)$, is $\hat{G}_{x y}(f)$ and the corresponding Kulite power spectra and far field spectra are $\hat{G}_{x x}(f)$ and $\hat{G}_{y y}(f)$.

$$
\begin{aligned}
\hat{G}_{x x}(f) & =\hat{G}_{s_{c} s_{c}}(f)+\hat{G}_{n_{1} n_{1}}(f) \\
\hat{G}_{y y}(f) & =\hat{G}_{s_{F} s_{F}}(f)+\hat{G} n_{2} n_{2}(f)
\end{aligned}
$$

These are obtained by averaging many ( approximately 467 ) successive directly calculated power spectral densities using a 20 second interval in most cases and a sampling rate of 48,000 samples per second. In practice, only estimates, $\hat{G}_{x y}(f)$ of $G_{x y}(f), \hat{G}_{x x}(f)$ of $G_{x x}(f)$, and $\hat{G}_{y y}(f)$ of $G_{y y}(f)$ can be obtained due to the finite observation interval and the^notation will be dropped.

The combustion noise reaching a microphone will be estimated using the magnitude squared coherence function, $\gamma_{x y}^{2}(f)$, where

$$
\gamma_{x y}^{2}(f)=\frac{\left|G_{x y}(f)\right|^{2}}{G_{x x}(f) G_{y y}(f)}
$$

Then

$$
\left|G_{x y}(f)\right|^{2}=\left|G_{s_{F} s_{c}}(f)\right|^{2}=|H(f)|^{2} G_{s_{c} s_{c}}^{2}(f)=G_{s_{F} s_{F}}(f) G_{s_{c} s_{c}}(f)
$$

and the coherent output power spectrum, $W_{s_{F} s_{F}}(f)$, is

$$
\begin{aligned}
W_{s_{F} s_{F}}(f) & =\gamma_{x y}^{2}(f) G_{y y}(f) \\
& =G_{s_{F} s_{F}}(f) \frac{G_{s_{c} s_{c}}(f)}{G_{s_{c} s_{c}}(f)+G_{n_{1} n_{1}}(f)}
\end{aligned}
$$

where we assume the extraneous noise terms are uncorrelated with each other and with the signals.

$$
G_{s_{c} n_{1}}(f)=G_{s_{F} n_{2}}(f)=G_{n_{1} n_{2}}(f)=0
$$

Thus the estimated coherent output power spectrum, $W_{s_{F} s_{F}}(f)=\gamma_{x y}^{2}(f) G_{y y}(f)$, will determine $G_{s_{F} s_{F}}(f)$ when the input noise is zero, $G_{n_{1} n_{1}}(f)=0$, regardless of the output noise $G_{n_{2} n_{2}}(f)$. In the frequency range of interest we may assume $G_{n_{1} n_{1}}(f)=0$ or just consider that $W_{s_{F} s_{F}}(f)$ is a measure of the combustion noise.

\section{Expected value of the spectrum}

In this section we outline the origin of the equation for $E\left[S_{x y}(f, T, K)\right]$ given in Eq. (23). As mentioned, a derivation of Eq. (23) is given by Bendat and Piersol ${ }^{14}$ section 3.2.3. A similar derivation is given by Davenport and Root ${ }^{53}$ page 106 and Jenkins and Watts ${ }^{18}$ section 6.2.1.

We have

$$
X_{k}(f, T)=\int_{0}^{T} x_{k}(t) \exp (-i 2 \pi f t) d t
$$




$$
\begin{aligned}
Y_{k}(f, T) & =\int_{0}^{T} y_{k}(t) \exp (-i 2 \pi f t) d t \\
S_{x y}(f, T, k) & =\frac{1}{T} X_{k}^{*}(f, T) Y_{k}(f, T)=\frac{1}{T} \int_{0}^{T} x_{k}(\alpha) \exp (+j 2 \pi f \alpha) d \alpha \int_{0}^{T} y_{k}(\beta) \exp (-j 2 \pi f \beta) d \beta \\
& =\frac{1}{T} \int_{0}^{T} \int_{0}^{T} x_{k}(\alpha) y_{k}(\beta) \exp (-j 2 \pi f(\beta-\alpha)) d \alpha d \beta
\end{aligned}
$$

Letting $\tau=\beta-\alpha$, the double integral becomes the sum of two integrals. The first corresponds to $\beta=0$ and the second corresponds to $\beta=T$. We also use the definition

$$
\begin{aligned}
R_{x y}(\tau)=E\left[x_{k}(\alpha) y_{k}(\alpha+\tau)\right] & \\
S_{x y}(f, T, k) & =\int_{-T}^{0}\left[\frac{1}{T} \int_{-\tau}^{T} x_{k}(\alpha) y_{k}(\alpha+\tau) d \alpha\right] \exp (-j 2 \pi f \tau) d \tau \\
& +\int_{0}^{T}\left[\frac{1}{T} \int_{0}^{T-\tau} x_{k}(\alpha) y_{k}(\alpha+\tau) d \alpha\right] \exp (-j 2 \pi f \tau) d \tau \\
E\left[S_{x y}(f, T, k)\right] & =E\left[\frac{1}{T} X_{k}^{*}(f, T) Y_{k}(f, T)\right] \\
& =\int_{-T}^{0}\left[\frac{1}{T} \int_{-\tau}^{T} R_{x y}(\tau) d \alpha\right] \exp (-j 2 \pi f \tau) d \tau \\
& +\int_{0}^{T}\left[\frac{1}{T} \int_{0}^{T-\tau} R_{x y}(\tau) d \alpha\right] \exp (-j 2 \pi f \tau) d \tau \\
& =\int_{-T}^{0}\left[1-\frac{\tau}{T}\right] R_{x y}(\tau) \exp (-j 2 \pi f \tau) d \tau+\int_{0}^{T}\left[1-\frac{\tau}{T}\right] R_{x y}(\tau) \exp (-j 2 \pi f \tau) d \tau \\
& =\int_{-T}^{T}\left[1-\frac{|\tau|}{T}\right] R_{x y}(\tau) \exp (-j 2 \pi f \tau) d \tau
\end{aligned}
$$

It should be noted that this expression indicates that the averaged periodogram procedure is an averaged truncated peridogram procedure which reduces the bias and variance in the original periodogram estimate by removing contributions from the "tails" and averaging the remainder over the record length, $T$ using the weighting function $1-\frac{|\tau|}{T}$. $\left(\right.$ Priestley $^{1}$ page 433 ). This averaged truncated peridogram creates a consistent estimate of the spectrum at each frequency. The original periodogram was devised for discrete spectra and works well for discrete spectra. It fails for continuous spectra where it can be shown the variance of the original periodogram for a Gaussian process does not go to zero. ( Davenport and Root ${ }^{53}$ page 107-108 , Koopmans ${ }^{55}$ Chapter 8, Priestley ${ }^{1}$ section 6.2.2,pages 429-432 ) 


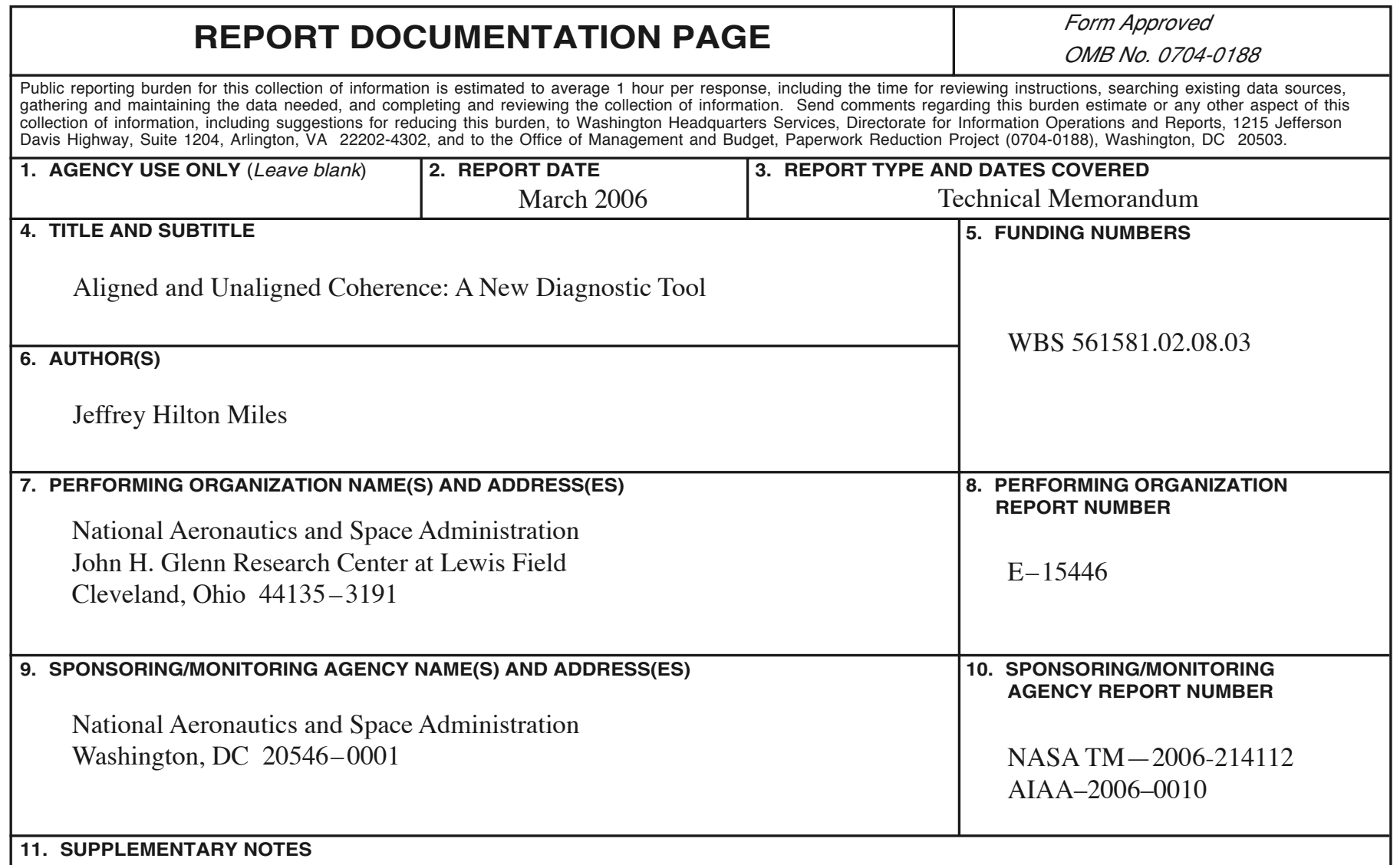

Prepared for the 44th Aerospace Sciences Meeting and Exhibit sponsored by the American Institute of Aeronautics and Astronautics, Reno, Nevada, January 9-12, 2006. Responsible person, Jeffrey Hilton Miles, organization code RTA, 216-433-5909.

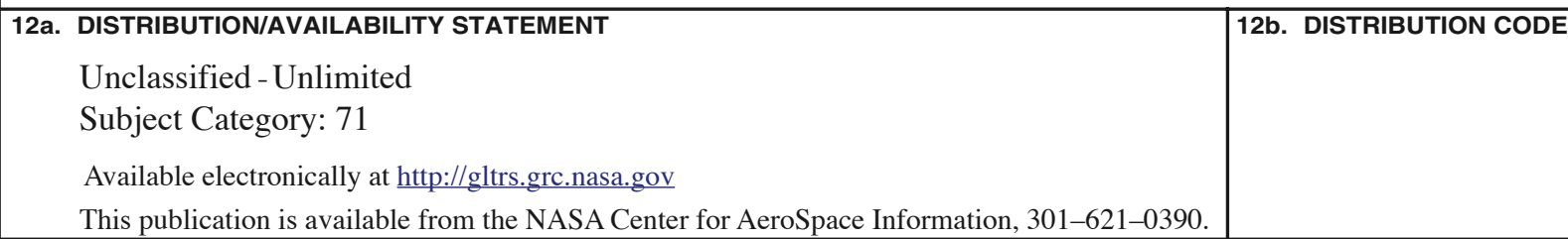

13. ABSTRACT (Maximum 200 words)

The study of combustion noise from turbofan engines has become important again as the noise from other sources like the fan and jet are reduced. A method has been developed to help identify combustion noise spectra using an aligned and unaligned coherence technique. When used with the well known three signal coherent power method and coherent power method it provides new information by separating tonal information from random process information. Examples are presented showing the underlying tonal structure which is buried under broadband noise and jet noise. The method is applied to data from a Pratt and Whitney PW4098 turbofan engine.

\begin{tabular}{|c|c|c|}
\hline \multicolumn{3}{|c|}{$\begin{array}{l}\text { Jet aircraft noise; Acoustic measurement; Combustion noise; Signal detection; Signal } \\
\text { analysis; Core noise }\end{array}$} \\
\hline $\begin{array}{l}\text { 17. SECURITY CLASSIFICATION } \\
\text { OF REPORT } \\
\text { Unclassified }\end{array}$ & $\begin{array}{l}\text { 18. SECURITY CLASSIFICATION } \\
\text { OF THIS PAGE }\end{array}$ & $\begin{array}{l}\text { 19. SECURITY CLASSIFICATION } \\
\text { OF ABSTRACT }\end{array}$ \\
\hline Unclassified & Unclassified & Unclassified \\
\hline
\end{tabular}



